\title{
NORM ESTIMATES FOR $\tau$-PSEUDODIFFERENTIAL OPERATORS IN WIENER AMALGAM AND MODULATION SPACES
}

\author{
ELENA CORDERO, LORENZA D'ELIA, AND S. IVAN TRAPASSO
}

\begin{abstract}
We study continuity properties on modulation spaces for $\tau$-pseudodifferential operators $\operatorname{Op}_{\tau}(a)$ with symbols $a$ in Wiener amalgam spaces. We obtain boundedness results for $\tau \in(0,1)$ whereas, in the end-points $\tau=0$ and $\tau=1$, the corresponding operators are in general unbounded. Furthermore, for $\tau \in(0,1)$, we exhibit a function of $\tau$ which is an upper bound for the operator norm. The continuity properties of $\operatorname{Op}_{\tau}(a)$, for any $\tau \in[0,1]$, with symbols $a$ in modulation spaces are well known. Here we find an upper bound for the operator norm which does not depend on the parameter $\tau \in[0,1]$, as expected.

Key ingredients are uniform continuity estimates for $\tau$-Wigner distributions.
\end{abstract}

\section{INTRODUCTION}

Pseudodifferential operators are mathematical tools used extensively in the theory of partial differential equations, engineering and quantum mechanics. Since their first appearance in the works by Kohn, Nirenberg [20] and Hörmander [19], they have been widely studied in the framework of classical analysis by plenty of authors, with privileged symbol classes being the so-called Hörmander class [19]. In this context, we also refer to the textbooks [23, 26, 28, 33].

Starting from the end of the 90's and during the last 20 years they have been considered in the context of time-frequency analysis. Many outcomes have been obtained, showing in particular that operators with rough symbols (functions not even differentiable or tempered distributions) may be bounded on $L^{2}\left(\mathbb{R}^{d}\right)$. The contributions are so many that we are not able to cite them all. See, for instance, $[2,5,8,10,16,17,18,21,22,25,27,29,30,31,32]$.

Assume $\tau \in[0,1], a \in \mathcal{S}^{\prime}\left(\mathbb{R}^{2 d}\right)$, then the $\tau$-pseudodifferential operator $\mathrm{Op}_{\tau}(a)$ with symbol $a$ can be defined by

$$
\mathrm{Op}_{\tau}(a) f(x)=\int_{\mathbb{R}^{d}} \int_{\mathbb{R}^{d}} e^{2 \pi i(x-y) \xi} a((1-\tau) x+\tau y, \xi) f(y) d y d \xi, \quad f \in \mathcal{S}\left(\mathbb{R}^{d}\right) .
$$

2010 Mathematics Subject Classification. 47G30,35S05,42B35,81S30.

Key words and phrases. $\tau$-Wigner distribution, $\tau$-pseudodifferential operators, Wiener amalgam spaces, modulation spaces. 
If $\tau=0$, the corresponding operator $\mathrm{Op}_{0}(a)$ is called the Kohn-Niremberg operator and can be rewritten as

$$
\mathrm{Op}_{0}(a) f(x)=\int_{\mathbb{R}^{d}} a(x, \xi) \hat{f}(\xi) e^{2 \pi i x \xi} d \xi, \quad f \in \mathcal{S}\left(\mathbb{R}^{d}\right)
$$

For $\tau=1 / 2, \mathrm{Op}_{1 / 2}(a)$ (proposed by Weyl in [34]) is called Weyl operator and takes the form

$$
\mathrm{Op}_{1 / 2}(a) f(x)=\int_{\mathbb{R}^{2 d}} a\left(\frac{x+y}{2}, \xi\right) f(y) e^{2 \pi i(x-y) \xi} d y d \xi, \quad f \in \mathcal{S}\left(\mathbb{R}^{d}\right) .
$$

In this paper we continue the study of boundedness properties of pseudodifferential operators using tools from time-frequency analysis. The main ingredients are the time-frequency representations related to the definition of $\tau$-pseudodifferential operators. For $\tau \in[0,1]$, the (cross- $) \tau$-Wigner distribution $(\tau$-WD) of signals $f, g \in L^{2}\left(\mathbb{R}^{d}\right)$ is defined by

$$
W_{\tau}(f, g)(x, \xi)=\int_{\mathbb{R}^{d}} e^{-2 \pi i t \xi} f(x+\tau t) \overline{g(x-(1-\tau) t)} d t, \quad(x, \xi) \in \mathbb{R}^{2 d} .
$$

For $f=g, W_{\tau} f:=W_{\tau}(f, f)$ is called the $\tau$-Wigner distribution of $f$. Note that $W_{\tau} f$ is a quadratic time-frequency representation which is a generalization of the well known Wigner distribution, recaptured in the case $\tau=1 / 2$ :

$$
W_{1 / 2}(f, g)(x, \xi)=W(f, g)(x, \xi)=\int_{\mathbb{R}^{d}} f\left(x+\frac{t}{2}\right) \overline{g\left(x-\frac{t}{2}\right)} e^{-2 \pi i t \xi} d t .
$$

For $\tau=0, W_{0}(f, g)$ is named (cross-)Rihaczek distribution

$$
W_{0}(f, g)(x, \xi)=\mathcal{R}(f, g)(x, \xi)=e^{-2 \pi i x \cdot \xi} f(x) \overline{\hat{g}(\xi)} ;
$$

and for $\tau=1, W_{1}(f, g)$ is the (cross-)conjugate Rihaczek distribution

$$
W_{1}(f, g)(x, \xi)=\mathcal{R}^{*}(f, g)(x, \xi)=\overline{\mathcal{R}(g, f)(x, \xi)}=e^{2 \pi i x \cdot \xi} \overline{g(x)} \hat{f}(\xi) .
$$

Given a symbol $a \in \mathcal{S}^{\prime}\left(\mathbb{R}^{2 d}\right)$, the $\tau$-pseudodifferential operator $\mathrm{Op}_{\tau}(a)$ in (1) can be defined weakly as a duality between the symbol $a$ and the $\tau$-WD $W_{\tau}(g, f)$ as follows

$$
\left\langle\mathrm{Op}_{\tau}(a) f, g\right\rangle=\left\langle a, W_{\tau}(g, f)\right\rangle, \quad f, g \in \mathcal{S}\left(\mathbb{R}^{d}\right) .
$$

Inspired by the work of Boulkhemair [5], we continue his investigation considering symbols in the new framework of Wiener amalgam spaces. Such spaces can be viewed as $L^{q}\left(L^{p}\right)$-norm of a time-frequency representation: the short-time Fourier transform (STFT) $V_{g} f$ of a signal $f \in \mathcal{S}^{\prime}\left(\mathbb{R}^{d}\right)$ with respect to a window function $g \in \mathcal{S}\left(\mathbb{R}^{d}\right)$, defined by

$$
V_{g} f(z)=\langle f, \pi(z) g\rangle=\mathcal{F}\left[f T_{x} g\right](\xi)=\int_{\mathbb{R}^{d}} f(t) \overline{g(t-x)} e^{-2 \pi i t \xi} d t
$$


for $z=(x, \xi) \in \mathbb{R}^{d} \times \mathbb{R}^{d}$. For simplicity, we recall their definition in the unweighted case, referring to the next section for a more general definition and related properties. A tempered distribution $f \in \mathcal{S}^{\prime}\left(\mathbb{R}^{d}\right)$ is in the Wiener amalgam space $W\left(\mathcal{F} L^{p}, L^{q}\right)\left(\mathbb{R}^{d}\right), 1 \leq p, q \leq \infty$, if

$$
\|f\|_{W\left(\mathcal{F} L^{p}, L^{q}\right)\left(\mathbb{R}^{d}\right)}:=\left(\int_{\mathbb{R}^{d}}\left(\int_{\mathbb{R}^{d}}\left|V_{g} f(x, \xi)\right|^{p} d \xi\right)^{q / p} d x\right)^{1 / q}<\infty .
$$

Roughly speaking, a distribution $f$ is in the space $W\left(\mathcal{F} L^{p}, L^{q}\right)\left(\mathbb{R}^{d}\right)$ if locally it behaves like a function in $\mathcal{F} L^{p}\left(\mathbb{R}^{d}\right)$ and globally decays as a function in $L^{q}\left(\mathbb{R}^{d}\right)$. Such spaces capture the different behaviour of functions/distributions on local and global levels. For instance, it can be shown that the delta distribution $\delta$ is in $W\left(\mathcal{F} L^{\infty}, L^{1}\right)\left(\mathbb{R}^{d}\right)$ : its Fourier transform $\mathcal{F} \delta=1$ belongs to $L^{\infty}\left(\mathbb{R}^{d}\right)$ and the compact support guarantees whatever decay at infinity.

Modulation spaces are closely related to such Wiener spaces. Indeed the modulation space $M^{p, q}\left(\mathbb{R}^{d}\right)$ can be defined by

$$
M^{p, q}\left(\mathbb{R}^{d}\right)=\mathcal{F}^{-1} W\left(\mathcal{F} L^{p}, L^{q}\right)\left(\mathbb{R}^{d}\right),
$$

where $\mathcal{F}^{-1}$ is the inverse Fourier transform.

Sufficient and necessary conditions for boundedness properties of pseudodifferential operators with symbols in modulation spaces and acting on the same spaces have been found in many papers, cf. $[6,7,31,32]$ and the bibliography therein. Here such conditions do not depend on the parameter $\tau \in[0,1]$, see Section 5 for details in this framework.

In our context we continue to study boundedness properties on modulation spaces but the symbols are in the Wiener ones. Here the continuity properties do depend on the parameter $\tau$.

For $1 \leq r_{1}, r_{2} \leq \infty$, we introduce the function

$$
\alpha_{\left(r_{1}, r_{2}\right)}(\tau)=\frac{1}{\tau^{d\left(\frac{1}{r_{1}^{\prime}}+\frac{1}{r_{2}}\right)}(1-\tau) d\left(\frac{1}{r_{1}}+\frac{1}{r_{2}^{\prime}}\right)}, \quad \tau \in(0,1) .
$$

Observe that the function $\alpha_{\left(r_{1}, r_{2}\right)}(\tau)$ is unbounded on $(0,1)$. Indeed, for $\left(r_{1}, r_{2}\right) \notin$ $\{(1, \infty),(\infty, 1)\}$,

$$
\lim _{\tau \rightarrow 0^{+}} \alpha_{\left(r_{1}, r_{2}\right)}(\tau)=\lim _{\tau \rightarrow 1^{-}} \alpha_{\left(r_{1}, r_{2}\right)}(\tau)=+\infty .
$$

For $\left(r_{1}, r_{2}\right)=(1, \infty)$ we have $\lim _{\tau \rightarrow 1^{-}} \alpha_{(1, \infty)}(\tau)=+\infty$ whereas, for $\left(r_{1}, r_{2}\right)=$ $(\infty, 1), \lim _{\tau \rightarrow 0^{+}} \alpha_{(\infty, 1)}(\tau)=+\infty$. An unweighted version of our main result, cf. Theorem 4.3 below, can be read as follows:

Theorem 1.1. Suppose that $1 \leq p, q, r_{1}, r_{2} \leq \infty$ satisfy

$$
q \leq p^{\prime}, \quad \max \left\{r_{1}, r_{2}, r_{1}^{\prime}, r_{2}^{\prime}\right\} \leq p .
$$


Let a be a symbol in $W\left(\mathcal{F} L^{p}, L^{q}\right)\left(\mathbb{R}^{2 d}\right)$. For $\tau \in(0,1)$, every $\tau$-pseudodifferential operator $\mathrm{Op}_{\tau}(a)$ is a bounded operator on $M^{r_{1}, r_{2}}\left(\mathbb{R}^{d}\right)$. Moreover, there exists a constant $C>0$ independent of $\tau$ such that

$$
\left\|\mathrm{Op}_{\tau}(a) f\right\|_{M^{r_{1}, r_{2}}} \leq C \alpha_{\left(r_{1}, r_{2}\right)}(\tau)\|a\|_{W\left(\mathcal{F} L^{p}, L^{q}\right)}\|f\|_{M^{r_{1}, r_{2}},}, \quad \tau \in(0,1) .
$$

Hence we have found an upper bound for the operator norm:

$$
\left\|\mathrm{Op}_{\tau}(a)\right\|_{\mathcal{B}\left(M^{\left.r_{1}, r_{2}\right)}\right.} \leq C \alpha_{\left(r_{1}, r_{2}\right)}(\tau)\|a\|_{W\left(\mathcal{F} L^{p}, L^{q}\right)}
$$

The unboundedness of the function $\alpha_{\left(r_{1}, r_{2}\right)}(\tau)$ in the end-points suggests that the boundedness results above fail in the case of Kohn-Nirenberg operators $\operatorname{Op}_{0}(a)$ and of operators with right symbol $\mathrm{Op}_{1}(a)$ (also called anti-Kohn-Nirenberg operators). Indeed, we exhibit precise counterexamples in Proposition 4.4 below.

The paper is organized as follows. Section 2 is focused on the preliminary definitions and properties of $\tau$-Wigner distributions and the involved function spaces. In Section 3, we study the continuity properties of $W_{\tau}(f, g)$ in the Wiener amalgam spaces, obtaining uniform estimates with respect to the parameter $\tau$. Section 4 is devoted to the proof of the main theorem: Theorem 4.3. We also treat the cases $\tau=0$ and $\tau=1$, showing examples of unbounded operators. Section 5 provides some useful remarks on the continuity results of $\mathrm{Op}_{\tau}(a)$ with symbol in modulation spaces.

Notation. We define the scalar product on $\mathbb{R}^{d}$ by $x y=x \cdot y$. The Schwartz class is denoted by $\mathcal{S}\left(\mathbb{R}^{d}\right)$ and its dual, the space of tempered distributions, by $\mathcal{S}^{\prime}\left(\mathbb{R}^{d}\right)$. The brackets $\langle\cdot, \cdot\rangle$ stand for the inner product on $L^{2}\left(\mathbb{R}^{d}\right)$ or for duality pairing between a tempered distribution in $\mathcal{S}^{\prime}$ and a function in $\mathcal{S}$ (for convention it is antilinear in the second argument).

We write $f \lesssim g$ to indicate $f(x) \leqslant C g(x)$ for every $x$ and some constant $C$, and similarly for $\gtrsim$. The notation $f \asymp g$ stands for $f \lesssim g$ and $f \gtrsim g$. We use a normalized Fourier transform

$$
\mathcal{F} f(\xi)=\int_{\mathbb{R}^{d}} e^{-2 \pi i x \xi} f(x) d x
$$

The translation operator $T_{x}$ of a function $f$ on $\mathbb{R}^{d}$ is defined as $T_{x} f(t)=f(t-x)$ and the modulation operator $M_{\xi} f(t)=e^{2 \pi i \xi t} f(t)$. For $z=(x, \xi)$, we denote the so-called time-frequency shift acting on a function or distribution as $\pi(z) f(t)=M_{\xi} T_{x} f(t)$. The reflection operator is defined as $\mathcal{I} f(x)=f(-x)$. For $1 \leq p \leq \infty$, the conjugate exponent $p^{\prime}$ of $p$ is the one that satisfies $1 / p+1 / p^{\prime}=1$. 


\section{Time-Frequency Representations and Function Spaces}

Denote by $J$ the canonical symplectic matrix in $\mathbb{R}^{2 d}$ :

$$
J=\left(\begin{array}{cc}
0_{d \times d} & I_{d \times d} \\
-I_{d \times d} & 0_{d \times d}
\end{array}\right) \in S p(2 d, \mathbb{R})
$$

where the symplectic group $S p(2 d, \mathbb{R})$ is defined by

$$
S p(d, \mathbb{R})=\left\{M \in G L(2 d, \mathbb{R}): M^{\top} J M=J\right\} .
$$

In the sequel we shall heavily use the following symplectic matrix

$$
\mathcal{A}_{\tau}=\left(\begin{array}{cc}
0_{d \times d} & \left(\frac{1-\tau}{\tau}\right)^{1 / 2} I_{d \times d} \\
-\left(\frac{\tau}{1-\tau}\right)^{1 / 2} I_{d \times d} & 0_{d \times d}
\end{array}\right), \quad \tau \in(0,1) .
$$

The main properties of $\mathcal{A}_{\tau}$ are detailed below. Their proof is attained by easy computations.

Lemma 2.1. For any $\tau \in(0,1)$, the matrix $\mathcal{A}_{\tau}$ in (11) enjoys the following properties:

(i) $\mathcal{A}_{\tau} \in S p(d, \mathbb{R})$; in particular, $\mathcal{A}_{1 / 2}=J$.

(ii) $\mathcal{A}_{\tau}^{\top}=-\mathcal{A}_{1-\tau}, \mathcal{A}_{\tau}^{-1}=-\mathcal{A}_{\tau}$.

(iii) $\mathcal{A}_{1-\tau} \mathcal{A}_{\tau}=\mathcal{A}_{\tau}^{\top} \mathcal{A}_{\tau}^{-1}=I_{2 d \times 2 d}-\mathcal{B}_{\tau}$, where

$$
\mathcal{B}_{\tau}=\left(\begin{array}{cc}
\frac{1}{1-\tau} I_{d \times d} & 0_{d \times d} \\
0_{d \times d} & \frac{1}{\tau} I_{d \times d}
\end{array}\right) .
$$

(iv) $\sqrt{\tau(1-\tau)}\left(\mathcal{A}_{\tau}+\mathcal{A}_{1-\tau}\right)=\sqrt{\tau(1-\tau)} \mathcal{B}_{\tau} \mathcal{A}_{\tau}=J$

2.1. $\tau$-Wigner Distributions and their Short-Time Fourier Transforms. We list now some useful features enjoyed by the $\tau$-WD which we will use later (cf. $[3,9])$.

Proposition 2.2. For $\tau \in[0,1], f, g, f_{i}, g_{i} \in L^{2}\left(\mathbb{R}^{d}\right), i=1,2$, we have

(i) $W_{1-\tau}(f, g)=\overline{W_{\tau}(g, f)}$.

(ii) $W_{\tau} f(x, \xi)=W_{1-\tau} \hat{f}(\xi,-x)$. Equivalently

$$
W_{\tau} \hat{f}(z)=W_{1-\tau} f(J z),
$$

where $J$ is the canonical symplectic matrix in (10).

(iii) Moyal's Formula for $\tau$-WD:

$$
\left\langle W_{\tau}\left(f_{1}, g_{1}\right), W_{\tau}\left(f_{2}, g_{2}\right)\right\rangle=\left\langle f_{1}, f_{2}\right\rangle \overline{\left\langle g_{1}, g_{2}\right\rangle} .
$$

(iv) Covariance property for the $\tau-W D$ :

$$
W_{\tau}(\pi(w) f, \pi(w) g)(z)=T_{w} W_{\tau}(f, g)(z)=W_{\tau}(f, g)(z-w), \quad w, z \in \mathbb{R}^{2 d} .
$$


To study continuity properties of the $\tau$-WD on modulation and Wiener spaces, we need to compute its Short-time Fourier transform (STFT). Recall that the STFT of a signal $f \in \mathcal{S}^{\prime}\left(\mathbb{R}^{d}\right)$ with respect a fixed window function $g \in \mathcal{S}\left(\mathbb{R}^{d}\right)$ is defined in (7). Important properties of STFT we shall use are as follows.

Proposition 2.1. For $f, f_{i}, g, g_{i} \in L^{2}\left(\mathbb{R}^{d}\right), i=1,2$, we have:

(i) Orthogonality relations for the STFT:

$$
\left\langle V_{g_{1}} f_{1}, V_{g_{2}} f_{2}\right\rangle_{L^{2}\left(\mathbb{R}^{2 d}\right)}=\left\langle f_{1}, f_{2}\right\rangle_{L^{2}\left(\mathbb{R}^{d}\right)}{\overline{\left\langle g_{1}, g_{2}\right\rangle_{L^{2}\left(\mathbb{R}^{d}\right)}}}_{.}
$$

(ii) STFT of time-frequency shifts:

$$
V_{M_{\omega} T_{u} g}\left(M_{\omega} T_{u} f\right)(x, \xi)=e^{2 \pi i(\omega x-\xi u)} V_{g} f(x, \xi), \quad u, x, \xi, \omega \in \mathbb{R}^{d} .
$$

(iii) For $g_{0}, g, \gamma \in \mathcal{S}\left(\mathbb{R}^{d}\right)$ such that $\langle\gamma, g\rangle \neq 0, f \in \mathcal{S}^{\prime}\left(\mathbb{R}^{d}\right)$,

$$
\left|V_{g_{0}} f(x, \xi)\right| \leq \frac{1}{|\langle\gamma, g\rangle|}\left(\left|V_{g} f\right| *\left|V_{g_{0}} \gamma\right|\right)(x, \xi), \quad(x, \xi) \in \mathbb{R}^{2 d} .
$$

(iv) Fundamental identity of time-frequency analysis:

$$
V_{g} f(x, \xi)=e^{-2 \pi i x \xi} V_{\hat{g}} \hat{f}(\xi,-x), \quad(x, \xi) \in \mathbb{R}^{2 d} .
$$

For $\tau \in(0,1)$, the $\tau$-Wigner distribution can be rephrased as a STFT, the key ingredient is the operator $A_{\tau}$ below.

Definition 2.2. For $\tau \in(0,1)$, we define the operator $A_{\tau}$ by

$$
A_{\tau}: f(t) \longmapsto \mathcal{I} f\left(\frac{1-\tau}{\tau} t\right) .
$$

Then (cf. [3, Lemma 6.2]):

Lemma 2.3. For $\tau \in(0,1), f, g \in L^{2}\left(\mathbb{R}^{d}\right)$, we have

$$
W_{\tau}(f, g)(x, \xi)=\frac{1}{\tau^{d}} e^{2 \pi i \frac{1}{\tau} x \xi} V_{A_{\tau} g} f\left(\frac{1}{1-\tau} x, \frac{1}{\tau} \xi\right), \quad(x, \xi) \in \mathbb{R}^{2 d} .
$$

The proof of the following lemma is a matter of computation.

Lemma 2.4. For $\tau \in(0,1), z=\left(z_{1}, z_{2}\right) \in \mathbb{R}^{2 d}$, the operators $A_{\tau}$ and $\pi(z)$ commute as follows

$$
\begin{aligned}
& \pi(z) A_{\tau}=A_{\tau} \pi\left(-\frac{1-\tau}{\tau} z_{1},-\frac{\tau}{1-\tau} z_{2}\right) \\
& A_{\tau} \pi(z)=\pi\left(-\frac{\tau}{1-\tau} z_{1},-\frac{1-\tau}{\tau} z_{2}\right) A_{\tau} .
\end{aligned}
$$

In the next lemmas we calculate the STFT of $W_{\tau}(g, f)$, generalizing [14, Lemma 4.3.1]. 
Lemma 2.5. Consider $\tau \in(0,1)$. Let $\varphi_{1}, \varphi_{2} \in \mathcal{S}\left(\mathbb{R}^{d}\right), f, g \in \mathcal{S}\left(\mathbb{R}^{d}\right)$ and set $\Phi_{\tau}=W_{\tau}\left(\varphi_{1}, \varphi_{2}\right)$. Then,

$V_{\Phi_{\tau}} W_{\tau}(g, f)(z, \zeta)=e^{-2 \pi i z_{2} \zeta_{2}} V_{\varphi_{1}} g\left(z_{1}-\tau \zeta_{2}, z_{2}+(1-\tau) \zeta_{1}\right) \overline{V_{\varphi_{2}} f\left(z_{1}+(1-\tau) \zeta_{2}, z_{2}-\tau \zeta_{1}\right)}$

where $z=\left(z_{1}, z_{2}\right), \zeta=\left(\zeta_{1}, \zeta_{2}\right) \in \mathbb{R}^{2 d}$.

Proof. Using the covariance property (14) and the representation of the $\tau$-Wigner distribution as a STFT in (20),

$$
\begin{aligned}
V_{\Phi_{\tau}} & W_{\tau}(g, f)(z, \zeta)=\left\langle W_{\tau}(g, f), M_{\zeta} T_{z} W_{\tau}\left(\varphi_{1}, \varphi_{2}\right)\right\rangle \\
& =\left\langle W_{\tau}(g, f), M_{\zeta} W_{\tau}\left(\pi(z) \varphi_{1}, \pi(z) \varphi_{2}\right)\right\rangle \\
& =\frac{1}{\tau^{2 d}} \int_{\mathbb{R}^{2 d}} V_{A_{\tau} f} g\left(\frac{1}{1-\tau} x, \frac{1}{\tau} \xi\right) e^{-2 \pi i(x, \xi) \cdot\left(\zeta_{1}, \zeta_{2}\right)} \overline{V_{A_{\tau} \pi(z) \varphi_{2}} \pi(z) \varphi_{1}\left(\frac{1}{1-\tau} x, \frac{1}{\tau} \xi\right)} d x d \xi \\
& =\frac{(1-\tau)^{d}}{\tau^{d}} \int_{\mathbb{R}^{2 d}} V_{A_{\tau} f} g(x, \xi) e^{-2 \pi i\left(\zeta_{1}, \zeta_{2}\right) \cdot((1-\tau) x, \tau \xi)} \overline{V_{A_{\tau} \pi(z) \varphi_{2}} \pi(z) \varphi_{1}(x, \xi)} d x d \xi .
\end{aligned}
$$

To shorten notation, we write

$$
c_{\tau}=\frac{(1-\tau)^{d}}{\tau^{d}} .
$$

Using formula (16), the orthogonality relations (15) and the commutation relations between $\pi$ and $A_{\tau}$ in Lemma 2.4, we compute

$$
\begin{aligned}
& V_{\Phi_{\tau}} W_{\tau}(g, f)(z, \zeta) \\
&=c_{\tau} \int_{\mathbb{R}^{2 d}} V_{\pi\left(\tau \zeta_{2},-(1-\tau) \zeta_{1}\right) A_{\tau} f} \pi\left(\tau \zeta_{2},-(1-\tau) \zeta_{1}\right) g \overline{V_{A_{\tau} \pi(z) \varphi_{2}} \pi(z) \varphi_{1}}(x, \xi) d x d \xi \\
&=c_{\tau}\left\langle\pi\left(\tau \zeta_{2},-(1-\tau) \zeta_{1}\right) g, \pi\left(z_{1}, z_{2}\right) \varphi_{1}\right\rangle \overline{\left\langle\pi\left(\tau \zeta_{2},-(1-\tau) \zeta_{1}\right) A_{\tau} f, A_{\tau} \pi\left(z_{1}, z_{2}\right) \varphi_{2}\right\rangle} \\
&=c_{\tau} e^{-2 \pi i \tau z_{2} \zeta_{2}}\langle g,\left.\pi\left(z_{1}-\tau \zeta_{2}, z_{2}+(1-\tau) \zeta_{1}\right) \varphi_{1}\right\rangle \\
& \times \overline{\left\langle A_{\tau} f, \pi\left(-\tau \zeta_{2},(1-\tau) \zeta_{1}\right) A_{\tau} \pi\left(z_{1}, z_{2}\right) \varphi_{2}\right\rangle} \\
&=c_{\tau} e^{-2 \pi i \tau z_{2} \zeta_{2}}\langle g,\left.\pi\left(z_{1}-\tau \zeta_{2}, z_{2}+(1-\tau) \zeta_{1}\right) \varphi_{1}\right\rangle \\
& \times \overline{\left\langle A_{\tau} f, A_{\tau} \pi\left((1-\tau) \zeta_{2},-\tau \zeta_{1}\right) \pi\left(z_{1}, z_{2}\right) \varphi_{2}\right\rangle} \\
&=e^{-2 \pi i \tau z_{2} \zeta_{2}} e^{-2 \pi i(1-\tau) z_{2} \zeta_{2}}\left\langle g, \pi\left(z_{1}-\tau \zeta_{2}, z_{2}+(1-\tau) \zeta_{1}\right) \varphi_{1}\right\rangle \\
& \quad \times \overline{\left\langle f, \pi\left(z_{1}+(1-\tau) \zeta_{2}, z_{2}-\tau \zeta_{1}\right) \varphi_{2}\right\rangle} \\
&=e^{-2 \pi i z_{2} \zeta_{2}} V_{\varphi_{1}} g\left(z_{1}-\tau \zeta_{2}, z_{2}+(1-\tau) \zeta_{1}\right) \overline{V_{\varphi_{2}} f\left(z_{1}+(1-\tau) \zeta_{2}, z_{2}-\tau \zeta_{1}\right)} .
\end{aligned}
$$

The claim is proved. 
Formula (23) can be equivalently written as $(24)$

$$
V_{\Phi_{\tau}} W_{\tau}(g, f)(z, \zeta)=e^{-2 \pi i z_{2} \zeta_{2}} V_{\varphi_{1}} g\left(z+\sqrt{\tau(1-\tau)} \mathcal{A}_{\tau}^{T} \zeta\right) \overline{V_{\varphi_{2}} f\left(z+\sqrt{\tau(1-\tau)} \mathcal{A}_{\tau} \zeta\right)}
$$

where $\mathcal{A}_{\tau}$ is a symplectic matrix defined in (11).

The previous lemma does not cover the case $\tau=0$ and $\tau=1$, which are treated below.

Lemma 2.6 (STFT of the Rihaczek distribution). Let $\varphi_{1}, \varphi_{2} \in \mathcal{S}\left(\mathbb{R}^{d}\right), f, g \in \mathcal{S}\left(\mathbb{R}^{d}\right)$ and set $\Phi_{0}=W_{0}\left(\varphi_{1}, \varphi_{2}\right)$. Then,

$$
V_{\Phi_{0}} W_{0}(g, f)(z, \zeta)=e^{-2 \pi i z_{2} \zeta_{2}} V_{\varphi_{1}} g\left(z_{1}, z_{2}+\zeta_{1}\right) \overline{V_{\varphi_{2}} f\left(z_{1}+\zeta_{2}, z_{2}\right)},
$$

where $z=\left(z_{1}, z_{2}\right), \zeta=\left(\zeta_{1}, \zeta_{2}\right) \in \mathbb{R}^{2 d}$.

Proof. We use the definition in (5) and formula (16) in the following computations:

$$
\begin{aligned}
V_{\Phi_{0}} & W_{0}(g, f)(z, \zeta)=\left\langle W_{0}(g, f), M_{\zeta} T_{z} W_{0}\left(\varphi_{1}, \varphi_{2}\right)\right\rangle \\
& =\int_{\mathbb{R}^{2 d}} e^{-2 \pi i x \xi} g(x) \overline{\hat{f}(\xi)} e^{-2 \pi i\left(x \zeta_{1}+\xi \zeta_{2}\right)} e^{2 \pi i\left(x-z_{1}\right)\left(\xi-z_{2}\right)} \overline{\varphi_{1}\left(x-z_{1}\right)} \widehat{\varphi_{2}}\left(\xi-z_{2}\right) d x d \xi \\
& =e^{2 \pi i z_{1} z_{2}} \int_{\mathbb{R}^{d}} e^{-2 \pi i x\left(z_{2}+\zeta_{1}\right)} g(x) \overline{\varphi_{1}\left(x-z_{1}\right)} d x \int_{\mathbb{R}^{d}} \overline{\hat{f}(\xi)} e^{-2 \pi i \xi\left(z_{1}+\zeta_{2}\right)} \widehat{\varphi_{2}}\left(\xi-z_{2}\right) d \xi \\
& =e^{2 \pi i z_{1} z_{2}} V_{\varphi_{1}} g\left(z_{1}, z_{2}+\zeta_{1}\right) \overline{V_{\widehat{\varphi_{2}}} \hat{f}\left(z_{2},-\left(z_{1}+\zeta_{2}\right)\right)} \\
& =e^{2 \pi i z_{1} z_{2}} V_{\varphi_{1}} g\left(z_{1}, z_{2}+\zeta_{1}\right) \overline{V_{\varphi_{2}} f\left(z_{1}+\zeta_{2}, z_{2}\right)} e^{-2 \pi i z_{2}\left(z_{1}+\zeta_{2}\right)} \\
& =e^{-2 \pi i z_{2} \zeta_{2}} V_{\varphi_{1}} g\left(z_{1}, z_{2}+\zeta_{1}\right) \overline{V_{\varphi_{2}} f\left(z_{1}+\zeta_{2}, z_{2}\right)}
\end{aligned}
$$

as desired.

Corollary 2.7 (STFT of the conjugate-Rihaczek distribution). Let $\varphi_{1}, \varphi_{2} \in \mathcal{S}\left(\mathbb{R}^{d}\right)$, $f, g \in \mathcal{S}\left(\mathbb{R}^{d}\right)$ and set $\Phi_{1}=W_{1}\left(\varphi_{1}, \varphi_{2}\right)$. Then,

$$
V_{\Phi_{1}} W_{1}(g, f)(z, \zeta)=e^{-2 \pi i z_{2} \zeta_{2}} V_{\varphi_{1}} g\left(z_{1}-\zeta_{2}, z_{2}\right) \overline{V_{\varphi_{2}} f\left(z_{1}, z_{2}-\zeta_{1}\right)}
$$

where $z=\left(z_{1}, z_{2}\right), \zeta=\left(\zeta_{1}, \zeta_{2}\right) \in \mathbb{R}^{2 d}$. 
Proof. Using the connection between the Rihaczek and the conjugate-Rihaczek distribution in (6) and the result of Lemma 2.6 we can write

$$
\begin{aligned}
& V_{\Phi_{1}} W_{1}(g, f)(z, \zeta)=\left\langle W_{1}(g, f), M_{\zeta} T_{z} W_{1}\left(\varphi_{1}, \varphi_{2}\right)\right\rangle \\
& =\left\langle\overline{W_{0}(f, g)}, M_{\zeta} T_{z} \overline{W_{0}\left(\varphi_{2}, \varphi_{1}\right)}\right\rangle \\
& =\overline{\left\langle W_{0}(f, g), M_{-\zeta} T_{z} W_{0}\left(\varphi_{2}, \varphi_{1}\right)\right\rangle} \\
& =\overline{V_{W_{0}\left(\varphi_{2}, \varphi_{1}\right)} W_{0}(f, g)(z,-\zeta)} \\
& =\overline{e^{2 \pi i z_{2} \zeta_{2}} V_{\varphi_{2}} f\left(z_{1}, z_{2}-\zeta_{1}\right) \overline{V_{\varphi_{1}} g\left(z_{1}-\zeta_{2}, z_{2}\right)}} \\
& =e^{-2 \pi i z_{2} \zeta_{2}} V_{\varphi_{1}} g\left(z_{1}-\zeta_{2}, z_{2}\right) \overline{V_{\varphi_{2}} f\left(z_{1}, z_{2}-\zeta_{1}\right)} \text {. }
\end{aligned}
$$

The proof is completed.

Remark 2.3. (i) Heuristically, formulae (25) and (26) can be inferred by putting $\tau=0$ and $\tau=1$ respectively in the expression (23).

(ii) The STFT of a multilinear version of the Rihaczek distribution was computed in [2, Lemma 3.3], cf. formula (3.3). However, there is a flaw in the phase factor of that formula. Indeed, the exponential $e^{2 \pi i u_{0} \cdot\left(u_{1}+\cdots+u_{m}\right)}$ should be replaced by $e^{2 \pi i \sum_{i=1}^{m} u_{i} \cdot v_{i}}$, as the linear case $m=1$ in (25) shows.

2.2. Generalized Gaussian Functions. In order to compute the norm of $W_{\tau}$ in Wiener amalgam spaces, generalized Gaussian functions will play a crucial role. Given $a, b, c>0$, the generalized Gaussian function is defined as

$$
f_{a, b, c}(x, \xi)=e^{-\pi a x^{2}} e^{-\pi b \xi^{2}} e^{2 \pi i c x \xi}, \quad(x, \xi) \in \mathbb{R}^{2 d} .
$$

In the sequel, we will employ the STFT of a generalized Gaussian function, computed in [6, Proposition 2.2]:

Proposition 2.4. For $\Phi(x, \xi)=e^{-\pi\left(x^{2}+\xi^{2}\right)}, z=\left(z_{1}, z_{2}\right), \zeta=\left(\zeta_{1}, \zeta_{2}\right) \in \mathbb{R}^{2 d}$, we obtain

$$
\begin{gathered}
V_{\Phi} f_{a, b, c}(z, \zeta)=C(a, b, c) e^{-\pi \frac{\left[a(b+1)+c^{2}\right] z_{1}^{2}+\left[(a+1) b+c^{2}\right] z_{2}^{2}+(b+1) \zeta_{1}^{2}+(a+1) \zeta_{2}^{2}-2 c\left(z_{1} \zeta_{2}+z_{2} \zeta_{1}\right)}{(a+1)(b+1)+c^{2}}} \\
\times e^{-\frac{2 \pi i}{a+1}\left[z_{1} \zeta_{1}+\left(c z_{1}-(a+1) \zeta_{2}\right) \frac{c \zeta_{1}+(a+1) z_{2}}{(a+1)(b+1)+c^{2}}\right]}
\end{gathered}
$$

with $C(a, b, c)=\left[(a+1)(b+1)+c^{2}\right]^{-d / 2}$.

The $\tau$-Wigner distribution of the Gaussian function $\varphi(t)=e^{-\pi t^{2}}$ is in turn a generalized Gaussian function, as showed in the next lemma.

Lemma 2.8. Consider $\varphi_{1}(t)=\varphi_{2}(t)=\varphi(t)=e^{-\pi t^{2}}, t \in \mathbb{R}^{d}$, and $\tau \in[0,1]$. Then

$$
W_{\tau} \varphi(x, \xi)=\frac{1}{\left(2 \tau^{2}-2 \tau+1\right)^{d / 2}} e^{-\pi \frac{1}{2 \tau^{2}-2 \tau+1} x^{2}} e^{-\pi \frac{1}{2 \tau^{2}-2 \tau+1} \xi^{2}} e^{2 \pi i \frac{2 \tau-1}{2 \tau^{2}-2 \tau+1} x \xi},
$$


for $\operatorname{all}(x, \xi) \in \mathbb{R}^{2 d}$.

Proof. Using the definition of the $\tau$-WD in (4),

$$
\begin{aligned}
W_{\tau} \varphi(x, \xi) & =\int_{\mathbb{R}^{d}} e^{-2 \pi i \xi t} e^{-\pi(x+\tau t)^{2}} e^{-\pi(x-(1-\tau) t)^{2}} d t \\
& =\int_{\mathbb{R}^{d}} e^{-2 \pi i \xi t} e^{-2 \pi x^{2}} e^{-\pi\left[\left(2 \tau^{2}-2 \tau+1\right) t^{2}+2(2 \tau-1) x t\right]} d t \\
& =e^{-2 \pi x^{2}+\pi\left(\frac{2 \tau-1}{\sqrt{2 \tau^{2}-2 \tau+1}}\right)^{2} x^{2}} \int_{\mathbb{R}^{d}} e^{-2 \pi i \xi t} e^{-\pi\left(\sqrt{2 \tau^{2}-2 \tau+1} t+\frac{2 \tau-1}{\sqrt{2 \tau^{2}-2 \tau+1}} x\right)^{2}} d t .
\end{aligned}
$$

We perform the following change of variables

$$
\sqrt{2 \tau^{2}-2 \tau+1} t+\frac{2 \tau-1}{\sqrt{2 \tau^{2}-2 \tau+1}} x=y,
$$

so that, naming $c(\tau)=2 \tau^{2}-2 \tau+1>0$,

$$
\begin{aligned}
W_{\tau} \varphi(x, \xi) & =\frac{1}{c(\tau)^{\frac{d}{2}}} e^{-\pi\left(2-\frac{(2 \tau-1)^{2}}{c(\tau)}\right) x^{2}} \int_{\mathbb{R}^{d}} e^{-2 \pi i \xi \frac{y}{\sqrt{c(\tau)}}} e^{2 \pi i \frac{2 \tau-1}{c(\tau)} \xi x} e^{-\pi y^{2}} d y \\
& =\frac{1}{c(\tau)^{\frac{d}{2}}} e^{-\pi \frac{1}{c(\tau)} x^{2}} e^{2 \pi i \frac{2 \tau-1}{c(\tau)} \xi x} e^{-\pi \frac{1}{c(\tau)} \xi^{2}}
\end{aligned}
$$

as desired.

2.3. Weights and Function Spaces. In time-frequency analysis, weight functions play an important role, since they describe the growth and the decay of a signal $f$ on the time-frequency plane $\mathbb{R}^{2 d}$. For a complete survey on weights, we refer to [15]. A weight function is a positive, locally integrable function on $\mathbb{R}^{2 d}$. In the sequel, we will need the following types of weight functions.

Definition 2.5. Let $v$ and $m$ be positive functions on $\mathbb{R}^{2 d}$.

(i) A weight $v$ is called submultiplicative if

$$
v\left(z_{1}+z_{2}\right) \leq v\left(z_{1}\right) v\left(z_{2}\right), \quad \forall z_{1}, z_{2} \in \mathbb{R}^{2 d} .
$$

(ii) Let $v$ be a submultiplicative weight, a positive function $m$ on $\mathbb{R}^{2 d}$ is called a $v$-moderate weight, if there exists a constant $C>0$, such that

$$
m\left(z_{1}+z_{2}\right) \leq C v\left(z_{1}\right) m\left(z_{2}\right), \quad \forall z_{1}, z_{2} \in \mathbb{R}^{2 d} .
$$

Let $\mathcal{M}_{v}\left(\mathbb{R}^{2 d}\right)$ be the space of all $v$-moderate weights. An important feature of submultiplicative weights is that they have at most an exponential growth, cf. [15, Lemma 4.2]): 
Lemma 2.9. If $v$ is submultiplicative and even weight, then there exist constants $C, a>0$ such that

$$
v(x) \leq C e^{a|x|}, \quad \forall x \in \mathbb{R}^{2 d} .
$$

From now on, we assume that $v$ is a continuous, positive, even, submultiplicative weight, i.e., $v(0)=1, v(z)=v(-z)$ and $v\left(z_{1}+z_{2}\right) \leq v\left(z_{1}\right) v\left(z_{2}\right)$, for all $z_{1}, z_{2} \in \mathbb{R}^{2 d}$. In what follows, we will use weight $1 / v$, which is a $v$-moderate weight:

$$
v(x)=v(x+y-y) \leq v(x+y) v(y) \quad \Rightarrow \quad \frac{1}{v(x+y)} \leq v(y) \frac{1}{v(x)} .
$$

Weight functions occur in the definition of general modulation spaces and Wiener amalgam spaces, where they offer a good device to measure a joint time-frequency concentration of a function or distribution. The definition of these function spaces relies on imposing a suitable norm on the short-time Fourier transform, defined in (7). For their basic properties we refer to $[11,12,13]$ and the textbooks $[9,14]$.

Given a non-zero window $g \in \mathcal{S}\left(\mathbb{R}^{d}\right)$, a $v$-moderate weight function $m$ on $\mathbb{R}^{2 d}$, $1 \leq p, q \leq \infty$, the modulation space $M_{m}^{p, q}\left(\mathbb{R}^{d}\right)$ consists of all tempered distributions $f \in \mathcal{S}^{\prime}\left(\mathbb{R}^{d}\right)$ such that $V_{g} f \in L_{m}^{p, q}\left(\mathbb{R}^{2 d}\right)$ (weighted mixed-norm spaces). The norm on $M_{m}^{p, q}\left(\mathbb{R}^{d}\right)$ is defined by

$$
\|f\|_{M_{m}^{p, q}}=\left\|V_{g} f\right\|_{L_{m}^{p, q}}=\left(\int_{\mathbb{R}^{d}}\left(\int_{\mathbb{R}^{d}}\left|V_{g} f(x, \xi)\right|^{p} m(x, \xi)^{p} d x\right)^{q / p} d \xi\right)^{1 / q}
$$

(obvious modifications for $p=\infty$ or $q=\infty$ ). If $p=q$, we write $M_{m}^{p}\left(\mathbb{R}^{d}\right)$ instead of $M_{m}^{p, p}\left(\mathbb{R}^{d}\right)$, and if $m(z) \equiv 1$ on $\mathbb{R}^{2 d}$, then we write $M^{p, q}\left(\mathbb{R}^{d}\right)$ and $M^{p}\left(\mathbb{R}^{d}\right)$ for $M_{m}^{p, q}\left(\mathbb{R}^{d}\right)$ and $M_{m}^{p, p}\left(\mathbb{R}^{d}\right)$.

The space $M_{m}^{p, q}\left(\mathbb{R}^{d}\right)$ is a Banach space whose definition is independent of the choice of the window $g$, in the sense that different non-zero window functions yield equivalent norms. The modulation space $M^{\infty, 1}\left(\mathbb{R}^{2 d}\right)$ is also called the Sjöstrand's class [24]. We recall the inclusion properties of modulation spaces. Suppose $m_{1}, m_{2}$ weight functions with $m_{2} \lesssim m_{1}$. Then, for $1 \leq p_{1}, p_{2}, q_{1}, q_{2} \leq \infty$, with $p_{1} \leq p_{2}$, $q_{1} \leq q_{2}$,

$$
\mathcal{S}\left(\mathbb{R}^{d}\right) \subseteq M_{m_{1}}^{p_{1}, q_{1}}\left(\mathbb{R}^{d}\right) \subseteq M_{m_{2}}^{p_{2}, q_{2}}\left(\mathbb{R}^{d}\right) \subseteq \mathcal{S}^{\prime}\left(\mathbb{R}^{d}\right)
$$

Note that for any $p, q \in[1, \infty]$ and any $m \in \mathcal{M}_{v}\left(\mathbb{R}^{2 d}\right)$, the inner product $\langle\cdot, \cdot\rangle$ on $\mathcal{S}\left(\mathbb{R}^{d}\right) \times \mathcal{S}\left(\mathbb{R}^{d}\right)$ extends to a continuous sesquilinear map $M_{m}^{p, q}\left(\mathbb{R}^{d}\right) \times M_{1 / m}^{p^{\prime}, q^{\prime}}\left(\mathbb{R}^{d}\right) \rightarrow \mathbb{C}$. Given even weigh functions $u, w$ on $\mathbb{R}^{d}$, the Wiener amalgam space $W\left(\mathcal{F} L_{u}^{p}, L_{w}^{q}\right)\left(\mathbb{R}^{d}\right)$ consist of all distributions $f \in \mathcal{S}^{\prime}\left(\mathbb{R}^{d}\right)$ such that

$$
\|f\|_{W\left(\mathcal{F} L_{u}^{p}, L_{w}^{q}\right)\left(\mathbb{R}^{d}\right)}:=\left(\int_{\mathbb{R}^{d}}\left(\int_{\mathbb{R}^{d}}\left|V_{g} f(x, \xi)\right|^{p} u^{p}(\xi) d \xi\right)^{q / p} w^{q}(x) d x\right)^{1 / q}<\infty
$$


where if $p=\infty$ or $q=\infty$, then we use the supremum norm.

The Wiener amalgam spaces $W\left(\mathcal{F} L_{u}^{p}, L_{w}^{q}\right)\left(\mathbb{R}^{d}\right)$ are the image of modulation spaces $M_{m}^{p, q}\left(\mathbb{R}^{d}\right)$ under the Fourier transform

$$
\mathcal{F}\left(M_{u \otimes w}^{p, q}\right)\left(\mathbb{R}^{d}\right)=W\left(\mathcal{F} L_{u}^{p}, L_{w}^{q}\right)\left(\mathbb{R}^{d}\right)
$$

Indeed, using Parseval identity in (7) and the fundamental identity (18), we can write $\left|V_{g} f(x, \xi)\right|=\left|V_{\hat{g}} \hat{f}(\xi,-x)\right|=\left|\mathcal{F}\left(\hat{f} T_{\xi} \overline{\hat{g}}\right)(-x)\right|$ and $($ recall $u(x)=u(-x))$

$$
\|f\|_{M_{u \otimes w}^{p, q}}=\left(\int_{\mathbb{R}^{d}}\left\|\hat{f} T_{\xi} \overline{\hat{g}}\right\|_{\mathcal{F} L_{u}^{p}}^{q} w^{q}(\xi) d \xi\right)^{1 / q}=\|\hat{f}\|_{W\left(\mathcal{F} L_{u}^{p}, L_{w}^{q}\right)} .
$$

Hence Wiener amalgam spaces are Banach spaces and their definition is independent of the choice of $g$.

Modulation and Wiener amalgam space norms of signals are weighted mixed-norm spaces of their short-time Fourier transforms. Hence their properties are based on those of the spaces $L_{m}^{p, q}$. Let us recall the convolution product of mixed-norm spaces [1]:

Lemma 2.10. For $1 \leq p_{i}, q_{i}, r, s \leq \infty, i=1,2, m \in \mathcal{M}_{v}\left(\mathbb{R}^{2 d}\right), F \in L_{v}^{p_{1}, q_{1}}\left(\mathbb{R}^{2 d}\right)$, $G \in L_{m}^{p_{2}, q_{2}}\left(\mathbb{R}^{2 d}\right)$, we have $F * G \in L_{m}^{r, s}\left(\mathbb{R}^{2 d}\right)$, with $1 / p_{1}+1 / p_{2}=1+1 / r, 1 / q_{1}+1 / q_{2}=$ $1+1 / s$ and

$$
\|F * G\|_{L_{m}^{r, s}} \leq\|F\|_{L_{v}^{p_{1}, q_{1}}}\|G\|_{L_{m}^{p_{2}, q_{2}}}
$$

We say that a measurable function $f$ on $\mathbb{R}^{4 d}$ is in the space $L_{z}^{\infty}\left(L_{\zeta, m}^{1}\right)\left(\mathbb{R}^{4 d}\right)$, with $m$ weight function on $\mathbb{R}^{2 d}$, if

$$
\|f\|_{L_{z}^{\infty}\left(L_{\zeta, m}^{1}\right)}=\sup _{z \in \mathbb{R}^{2 d}} \int_{\mathbb{R}^{2 d}}|f(z, \zeta)| m(\zeta) d \zeta<\infty
$$

When working on the STFT of $\tau$-WD, we will use the following Young-type inequality:

Lemma 2.11. If $m \in \mathcal{M}_{v}\left(\mathbb{R}^{2 d}\right)$, $f \in L_{1 \otimes v}^{1}\left(\mathbb{R}^{4 d}\right)$ and $g \in L_{z}^{\infty}\left(L_{\zeta, m}^{1}\right)\left(\mathbb{R}^{4 d}\right)$, then $f * g \in L_{z}^{\infty}\left(L_{\zeta, m}^{1}\right)\left(\mathbb{R}^{4 d}\right)$, with

$$
\|f * g\|_{L_{z}^{\infty}\left(L_{\zeta, m}^{1}\right)} \leq\|f\|_{L_{1 \otimes v}^{1}}\|g\|_{L_{z}^{\infty}\left(L_{\zeta, m}^{1}\right)} .
$$


Proof. Using the definition of $L_{z}^{\infty}\left(L_{\zeta, m}^{1}\right)$-norm in (32),

$$
\begin{aligned}
I:=\|f * g\|_{L_{z}^{\infty}\left(L_{\zeta, m}^{1}\right)} & =\sup _{z \in \mathbb{R}^{2 d}} \int_{\mathbb{R}^{2 d}}|f * g|(z, \zeta) m(\zeta) d \zeta \\
& =\sup _{z \in \mathbb{R}^{2 d}} \int_{\mathbb{R}^{2 d}}\left|\int_{\mathbb{R}^{4 d}} f(y, \eta) g(z-y, \zeta-\eta) d y d \eta\right| m(\zeta) d \zeta \\
& \leq \sup _{z \in \mathbb{R}^{2 d}} \int_{\mathbb{R}^{2 d}} \int_{\mathbb{R}^{2 d}}\left(\int_{\mathbb{R}^{2 d}}|f|(y, \eta)|g|(z-y, \zeta-\eta) d \eta\right) m(\zeta) d y d \zeta \\
& =\sup _{z \in \mathbb{R}^{2 d}} \int_{\mathbb{R}^{2 d}} \int_{\mathbb{R}^{2 d}}(|f|(y, \cdot) *|g|(z-y, \cdot))(\zeta) m(\zeta) d y d \zeta .
\end{aligned}
$$

By Young's inequality (31),

$$
\begin{aligned}
I & =\sup _{z \in \mathbb{R}^{2 d}} \int_{\mathbb{R}^{2 d}}\||f|(y, \cdot)\|_{L_{v}^{1}}\||g|(z-y, \cdot)\|_{L_{m}^{1}} d y \\
& \leq \int_{\mathbb{R}^{2 d}}\||f|(y, \cdot)\|_{L_{v}^{1}} \sup _{z \in \mathbb{R}^{d}}\||g|(z-y, \cdot)\|_{L_{m}^{1}} d y \\
& =\|g\|_{L_{z}^{\infty}\left(L_{\zeta, m}^{1}\right)}\|f\|_{L_{1 \otimes v}^{1}},
\end{aligned}
$$

as claimed.

A particular case of Lemma 2.10 gives:

Lemma 2.12. Suppose $m \in \mathcal{M}_{v}\left(\mathbb{R}^{2 d}\right), f \in L_{1 \otimes v}^{1}\left(\mathbb{R}^{4 d}\right)$ and $g \in L_{1 \otimes m}^{2}\left(\mathbb{R}^{4 d}\right)$. Then $f * g \in L_{1 \otimes m}^{2}\left(\mathbb{R}^{4 d}\right)$, with

$$
\|f * g\|_{L_{1 \otimes m}^{2}} \leq\|f\|_{L_{1 \otimes v}^{1}}\|g\|_{L_{1 \otimes m}^{2}} .
$$

\section{Boundedness properties of $\tau$-Wigner Distributions}

This section is devoted to investigate the continuity properties of $\tau$-Wigner distributions in the realm of Wiener and modulation spaces. For a submultiplicative weight $v$, we set

$$
v_{J}(z)=v(J z)
$$

where $J$ denotes the canonical symplectic matrix (10).

Lemma 3.1. Assume that $m \in \mathcal{M}_{v}\left(\mathbb{R}^{2 d}\right), 1 \leq p_{1}, p_{2} \leq \infty, f \in M_{m}^{p_{1}, p_{2}}\left(\mathbb{R}^{d}\right)$, $g \in M_{1 / m}^{p_{1}^{\prime}, p_{2}^{\prime}}\left(\mathbb{R}^{d}\right)$. Then for every $\tau \in(0,1)$, the $\tau$-Wigner distribution $W_{\tau}(g, f)$ is in $W\left(\mathcal{F} L_{1 / v_{J}}^{1}, L^{\infty}\right)\left(\mathbb{R}^{2 d}\right)$, with

$$
\left\|W_{\tau}(g, f)\right\|_{W\left(\mathcal{F} L_{1 / v_{J}}^{1}, L^{\infty}\right)} \leq C \alpha_{\left(p_{1}, p_{2}\right)}(\tau)\|f\|_{M_{m}^{p_{1}, p_{2}}}\|g\|_{M_{1 / m}^{p_{1}^{\prime}, p_{2}^{\prime}}}
$$

where the function $\alpha_{\left(p_{1}, p_{2}\right)}(\tau)$ is defined in (8) and $C>0$ is independent of $\tau$. 
Proof. We compute the STFT of $W_{\tau}(g, f)$ with respect to the window function $\Phi_{\tau} \in \mathcal{S}\left(\mathbb{R}^{2 d}\right)$ defined in Lemma 2.5. Using that lemma and the properties of the matrix $\mathcal{A}_{\tau}$ in Lemma 2.1 , by performing the change of variables $\sqrt{\tau(1-\tau)} \mathcal{A}_{\tau} \zeta=\eta$, we deduce

$$
\begin{aligned}
& \int_{\mathbb{R}^{2 d}}\left|V_{\Phi_{\tau}} W_{\tau}(g, f)\right|(z, \zeta) \frac{1}{v(J \zeta)} d \zeta \\
& =\int_{\mathbb{R}^{2 d}}\left|V_{\varphi_{1}} g\left(z+\sqrt{\tau(1-\tau)} \mathcal{A}_{\tau}^{T} \zeta\right)\right|\left|V_{\varphi_{2}} f\left(z+\sqrt{\tau(1-\tau)} \mathcal{A}_{\tau} \zeta\right)\right| \frac{1}{v\left(\sqrt{\tau(1-\tau)} \mathcal{B}_{\tau} \mathcal{A}_{\tau} \zeta\right)} d \zeta \\
& =\frac{1}{[\tau(1-\tau)]^{d}} \int_{\mathbb{R}^{2 d}}\left|V_{\varphi_{1}} g\left(z+\mathcal{A}_{1-\tau} \mathcal{A}_{\tau} \eta\right)\right|\left|V_{\varphi_{2}} f(z+\eta)\right| \frac{1}{v\left(\mathcal{B}_{\tau} \eta\right)} d \eta
\end{aligned}
$$

Since $m$ is a $v$-moderate weight, we can find a positive constant $C$, independent of $\tau$, such that

$$
\frac{1}{v\left(\mathcal{B}_{\tau} \eta\right)} \leq C \frac{m(z+\eta)}{m\left(z+\mathcal{A}_{1-\tau} \mathcal{A}_{\tau} \eta\right)}
$$

so that

$$
\begin{aligned}
& \int_{\mathbb{R}^{2 d}}\left|V_{\Phi_{\tau}} W_{\tau}(g, f)\right|(z, \zeta) \frac{1}{v(J \zeta)} d \zeta \\
& \quad \leq C \frac{1}{[\tau(1-\tau)]^{d}} \int_{\mathbb{R}^{2 d}}\left|V_{\varphi_{1}} g\left(z+\mathcal{A}_{1-\tau} \mathcal{A}_{\tau} \eta\right)\right|\left|V_{\varphi_{2}} f(z+\eta)\right| \frac{m(z+\eta)}{m\left(z+\mathcal{A}_{1-\tau} \mathcal{A}_{\tau} \eta\right)} d \eta
\end{aligned}
$$

Consequently,

$$
\begin{aligned}
& \left\|W_{\tau}(g, f)\right\|_{W\left(\mathcal{F} L_{1 / v_{J}}^{1}, L^{\infty}\right)} \\
& \asymp \sup _{z \in \mathbb{R}^{2 d}} \int_{\mathbb{R}^{2 d}}\left|V_{\varphi_{1}} g\left(z+\sqrt{\tau(1-\tau)} \mathcal{A}_{\tau}^{T} \zeta\right)\right|\left|V_{\varphi_{2}} f\left(z+\sqrt{\tau(1-\tau)} \mathcal{A}_{\tau} \zeta\right)\right| \frac{1}{v\left(\sqrt{\tau(1-\tau)} \mathcal{B}_{\tau} \mathcal{A}_{\tau} \zeta\right)} d \zeta \\
& \leq C \frac{1}{[\tau(1-\tau)]^{d}} \sup _{z \in \mathbb{R}^{2 d}} \int_{\mathbb{R}^{2 d}}\left|V_{\varphi_{1}} g\left(z+\mathcal{A}_{1-\tau} \mathcal{A}_{\tau} \eta\right)\right|\left|V_{\varphi_{2}} f(z+\eta)\right| \frac{m(z+\eta)}{m\left(z+\mathcal{A}_{1-\tau} \mathcal{A}_{\tau} \eta\right)} d \eta \\
& \leq C \frac{1}{[\tau(1-\tau)]^{d}}\left\|V_{\varphi_{1}} f m\right\|_{L^{p_{1}, p_{2}}}\left\|V_{\varphi_{2}} g \frac{1}{m}\left(z+\mathcal{A}_{1-\tau} \mathcal{A}_{\tau} \cdot\right)\right\|_{L^{p_{1}^{\prime}, p_{2}^{\prime}}}
\end{aligned}
$$

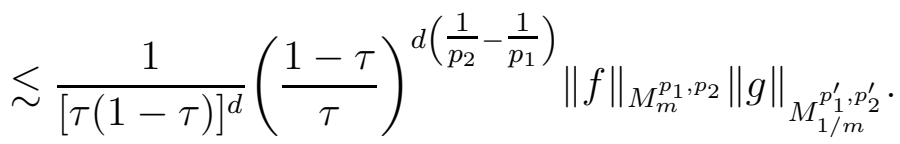

The claim is proved.

The previous estimate is not uniform with respect to $\tau$, in the sense that the $W\left(\mathcal{F} L_{1 / v_{J}}^{1}, L^{\infty}\right)$-norm of the $\tau$-WD has been calculated by using a window function $\Phi_{\tau}$ depending on $\tau$. The next goal is to find an upper bound of this norm independent of $\tau$. We will need the following result. 
Lemma 3.2. Consider $\Phi(x, \xi)=e^{-\pi\left(x^{2}+\xi^{2}\right)},(x, \xi) \in \mathbb{R}^{2 d}$, and $\Phi_{\tau}=W_{\tau}(\varphi, \varphi)$, where $\varphi(t)=e^{-\pi t^{2}}, t \in \mathbb{R}^{d}$. Then, for $v_{J}$ in (33), there exists a constant $C>0$ such that

$$
\left\|V_{\Phi} \Phi_{\tau}\right\|_{L_{1 \otimes v_{J}}^{1}} \leq C, \quad \forall \tau \in[0,1]
$$

Consequently,

$$
\left\|\Phi_{\tau}\right\|_{M_{1 \otimes v_{J}}^{1}} \leq C, \quad \forall \tau \in[0,1] .
$$

Proof. Using Lemma 2.8 and formula (28), with $z=\left(z_{1}, z_{2}\right), \zeta=\left(\zeta_{1}, \zeta_{2}\right) \in \mathbb{R}^{2 d}$, we compute

$$
\begin{aligned}
& \left|V_{\Phi} \Phi_{\tau}\right|(z, \zeta)=\frac{1}{\left(2 \tau^{2}-2 \tau+1\right)^{d / 2}} \frac{\left(2 \tau^{2}-2 \tau+1\right)^{d / 2}}{\left(2 \tau^{2}-2 \tau+5\right)^{d / 2}} \\
& \times e^{-\pi \frac{\frac{3}{2 \tau^{2}-2 \tau+1}\left(z_{1}^{2}+z_{2}^{2}\right)+\frac{2 \tau^{2}-2 \tau+2}{2 \tau^{2}-2 \tau+1}\left(\zeta_{1}^{2}+\zeta_{2}^{2}\right)-2 \frac{2 \tau-1}{2 \tau^{2}-2 \tau+1}\left(z_{1} \zeta_{2}+z_{2} \zeta_{1}\right)}{\frac{2 \tau^{2}-2 \tau+5}{2 \tau^{2}-2 \tau+1}}} \\
& =\frac{1}{\left(2 \tau^{2}-2 \tau+5\right)^{d / 2}} e^{-\pi \frac{3\left(z_{1}^{2}+z_{2}^{2}\right)+\left(2 \tau^{2}-2 \tau+2\right)\left(\zeta_{1}^{2}+\zeta_{2}^{2}\right)+(2-4 \tau)\left(z_{1} \zeta_{2}+z_{2} \zeta_{1}\right)}{2 \tau^{2}-2 \tau+5}} .
\end{aligned}
$$

Observing that

$$
\frac{1}{\left(2 \tau^{2}-2 \tau+5\right)^{d / 2}} \leq \max _{\tau \in(0,1)} \frac{1}{\left(2 \tau^{2}-2 \tau+5\right)^{d / 2}}=\left(\frac{2}{9}\right)^{d / 2}
$$

by Lemma 2.9, we have,

$$
\begin{aligned}
\left\|V_{\Phi} \Phi_{\tau}\right\|_{L_{1 \otimes v_{J}}} \leq & \left(\frac{2}{9}\right)^{d / 2} \\
& \times \int_{\mathbb{R}^{2 d}} \int_{\mathbb{R}^{2 d}} e^{-\pi \frac{3\left(z_{1}^{2}+z_{2}^{2}\right)+\left(2 \tau^{2}-2 \tau+2\right)\left(\zeta_{1}^{2}+\zeta_{2}^{2}\right)+(2-4 \tau)\left(z_{1} \zeta_{2}+z_{2} \zeta_{1}\right)}{2 \tau^{2}-2 \tau+5}} v_{J}(\zeta) d \zeta_{1} d \zeta_{2} d z_{1} d z_{2} \\
\leq & C \int_{\mathbb{R}^{2 d}} \int_{\mathbb{R}^{2 d}} e^{-\pi \frac{3\left(z_{1}^{2}+z_{2}^{2}\right)+\left(2 \tau^{2}-2 \tau+2\right)\left(\zeta_{1}^{2}+\zeta_{2}^{2}\right)+(2-4 \tau)\left(z_{1} \zeta_{2}+z_{2} \zeta_{1}\right)}{2 \tau^{2}-2 \tau+5}} e^{a|J \zeta|} d \zeta_{1} d \zeta_{2} d z_{1} d z_{2} \\
= & C \int_{\mathbb{R}^{2 d}} e^{-\pi \frac{3\left(z_{1}^{2}+z_{2}^{2}\right)}{2 \tau^{2}-2 \tau+5}} I_{1} d z_{1} d z_{2},
\end{aligned}
$$

where

$$
I_{1}:=\int_{\mathbb{R}^{2 d}} e^{-\pi \frac{\left(2 \tau^{2}-2 \tau+2\right)\left(\zeta_{1}^{2}+\zeta_{2}^{2}\right)+(2-4 \tau)\left(z_{1} \zeta_{2}+z_{2} \zeta_{1}\right)}{2 \tau^{2}-2 \tau+5}} e^{a|J \zeta|} d \zeta_{1} d \zeta_{2} .
$$


The integral $I_{1}$ can be computed as follows

$$
\begin{aligned}
I_{1} & =\int_{\mathbb{R}^{2 d}} e^{-\pi \frac{\left(2 \tau^{2}-2 \tau+2\right)\left(\zeta_{1}^{2}+\zeta_{2}^{2}\right)+(2-4 \tau)\left(z_{1} \zeta_{2}+z_{2} \zeta_{1}\right)}{2 \tau^{2}-2 \tau+5}} e^{a|J \zeta|} d \zeta_{1} d \zeta_{2} \\
& \leq \int_{\mathbb{R}^{2 d}} e^{-\pi \frac{\left(2 \tau^{2}-2 \tau+2\right)\left(\zeta_{1}^{2}+\zeta_{2}^{2}\right)+(2-4 \tau)\left(z_{1} \zeta_{2}+z_{2} \zeta_{1}\right)}{2 \tau^{2}-2 \tau+5}} e^{a\left(\left|\zeta_{1}\right|+\left|\zeta_{2}\right|\right)} d \zeta_{1} d \zeta_{2} \\
& =\left(\int_{\mathbb{R}^{d}} e^{-\pi \frac{\left(2 \tau^{2}-2 \tau+2\right) \zeta_{1}^{2}+(2-4 \tau) z_{2} \zeta_{1}}{2 \tau^{2}-2 \tau+5}} e^{a\left|\zeta_{1}\right|} d \zeta_{1}\right)\left(\int_{\mathbb{R}^{d}} e^{-\pi \frac{\left(2 \tau^{2}-2 \tau+2\right) \zeta_{2}^{2}+(2-4 \tau) z_{1} \zeta_{2}}{2 \tau^{2}-2 \tau+5}} e^{a\left|\zeta_{2}\right|} d \zeta_{2}\right) .
\end{aligned}
$$

We calculate the integral with respect to the variable $\zeta_{1}$ (the other integral is analogous):

$$
\begin{aligned}
& \int_{\mathbb{R}^{d}} e^{-\pi \frac{\left(2 \tau^{2}-2 \tau+2\right) \zeta_{1}^{2}+(2-4 \tau) z_{2} \zeta_{1}}{2 \tau^{2}-2 \tau+5}} e^{a\left|\zeta_{1}\right|} d \zeta_{1}=\int_{\mathbb{R}^{d}} e^{-\pi \frac{\left(2 \tau^{2}-2 \tau+2\right) \zeta_{1}^{2}+(2-4 \tau) z_{2} \zeta_{1}+\frac{(1-2 \tau)^{2} z_{2}^{2}}{2 \tau^{2}-2 \tau+2}-\frac{(1-2 \tau)^{2} z_{2}^{2}}{2 \tau^{2}-2 \tau+2}}{2 \tau^{2}-2 \tau+5}} e^{a\left|\zeta_{1}\right|} d \zeta_{1} \\
& =e^{\pi \frac{(1-2 \tau)^{2} z_{2}^{2}}{\left(2 \tau^{2}-2 \tau+2\right)\left(2 \tau^{2}-2 \tau+5\right)}} \\
& \times \int_{\mathbb{R}^{d}} e^{-\pi \frac{\left(\sqrt{2 \tau^{2}-2 \tau+2} \zeta_{1}+\frac{1-2 \tau}{\sqrt{2 \tau^{2}-2 \tau+2}} z_{2}\right)^{2}}{2 \tau^{2}-2 \tau+5}} e^{a\left|\zeta_{1}\right|} d \zeta_{1} \\
& =e^{\pi \frac{(1-2 \tau)^{2} z_{2}^{2}}{\left(2 \tau^{2}-2 \tau+2\right)\left(2 \tau^{2}-2 \tau+5\right)}} \underbrace{\int_{\mathbb{R}^{d}} e^{-\pi \frac{\left(\left(2 \tau^{2}-2 \tau+2\right) \zeta_{1}+(1-2 \tau) z_{2}\right)^{2}}{\left(2 \tau^{2}-2 \tau+5\right)\left(2 \tau^{2}-2 \tau+2\right)}} e^{a\left|\zeta_{1}\right|} d \zeta_{1}}_{:=I_{3}} .
\end{aligned}
$$

In $I_{3}$ we perform the following change of variables

$$
\left(2 \tau^{2}-2 \tau+2\right) \zeta_{1}+(1-2 \tau) z_{2}=\eta_{1}
$$

so that,

$$
\begin{aligned}
I_{3} & =\frac{1}{\left(2 \tau^{2}-2 \tau+2\right)^{d}} \int_{\mathbb{R}^{d}} e^{-\pi \frac{\eta_{1}^{2}}{\left(2 \tau^{2}-2 \tau+5\right)\left(2 \tau^{2}-2 \tau+2\right)}} e^{\frac{a}{2 \tau^{2}-2 \tau+2}\left|\eta_{1}-(1-2 \tau) z_{2}\right|} d \eta_{1} \\
& \leq C_{1}^{d} e^{\frac{a|1-2 \tau|}{2 \tau^{2}-2 \tau+2}\left|z_{2}\right|} \int_{\mathbb{R}^{d}} e^{-\pi C_{2} \eta_{1}^{2}} e^{a C_{1}\left|\eta_{1}\right|} d \eta_{1},
\end{aligned}
$$

where

$$
C_{1}=\max _{\tau \in[0,1]} \frac{1}{\left(2 \tau^{2}-2 \tau+2\right)}=\frac{2}{3}, \quad C_{2}=\min _{\tau \in[0,1]} \frac{1}{\left(2 \tau^{2}-2 \tau+5\right)\left(2 \tau^{2}-2 \tau+2\right)}=\frac{1}{10} .
$$


Using $\lim _{\left|\eta_{1}\right| \rightarrow \infty} e^{-\pi \frac{C_{1}}{2} \eta_{1}^{2}} e^{a C_{2}\left|\eta_{1}\right|}=0$, for every $\epsilon>0$ there exists $R>0$ such that $e^{-\pi \frac{C_{1}}{2} \eta_{1}^{2}} e^{a C_{2}\left|\eta_{1}\right|} \leq \epsilon$, for all $\left|\eta_{1}\right|$ with $\left|\eta_{1}\right|>R$. Hence

$$
\begin{aligned}
I_{3} & \leq C_{1}^{d} e^{\frac{a|1-2 \tau|}{2 \tau^{2}-2 \tau+2}\left|z_{2}\right|} \int_{\mathbb{R}^{d}} e^{-\pi C_{2} \eta_{1}^{2}} e^{a C_{1}\left|\eta_{1}\right|} d \eta_{1} \\
& =C_{1}^{d} e^{\frac{a|1-2 \tau|}{2 \tau^{2}-2 \tau+2}\left|z_{2}\right|}\left(\int_{\left\{\eta_{1} \in \mathbb{R}^{d}:\left|\eta_{1}\right| \leq R\right\}} e^{-\pi C_{2} \eta_{1}^{2}} e^{a C_{1}\left|\eta_{1}\right|} d \eta_{1}+\int_{\left\{\eta_{1} \in \mathbb{R}^{d}:\left|\eta_{1}\right|>R\right\}} e^{-\pi C_{2} \eta_{1}^{2}} e^{a C_{1}\left|\eta_{1}\right|} d \eta_{1}\right) \\
& \leq C_{1}^{d} e^{\frac{a|1-2 \tau|}{2 \tau^{2}-2 \tau+2}\left|z_{2}\right|}\left(e^{a C_{1} R} \int_{\mathbb{R}^{d}} e^{-\pi C_{2} \eta_{1}^{2}} d \eta_{1}+\epsilon \int_{\mathbb{R}^{d}} e^{-\pi \frac{C_{2}}{2} \eta_{1}^{2}} d \eta_{1}\right)=\tilde{C} e^{\frac{a|1-2 \tau|}{2 \tau^{2}-2 \tau+2}\left|z_{2}\right|}<\infty,
\end{aligned}
$$

where $\tilde{C}$ is a constant independent of $\tau$. In conclusion, the integral $I_{1}$ can be majorized as

$$
I_{1} \leq 2 \tilde{C} e^{\pi \frac{(1-2 \tau)^{2} z_{2}^{2}}{\left(2 \tau^{2}-2 \tau+2\right)\left(2 \tau^{2}-2 \tau+5\right)}+\frac{a|1-2 \tau|}{2 \tau^{2}-2 \tau+2}\left|z_{2}\right|} e^{\pi \frac{(1-2 \tau)^{2} z_{1}^{2}}{\left(2 \tau^{2}-2 \tau+2\right)\left(2 \tau^{2}-2 \tau+5\right)}+\frac{a|1-2 \tau|}{2 \tau^{2}-2 \tau+2}\left|z_{1}\right|}
$$

Thus, there exists a constant $M_{1}>0$ independent of $\tau$ such that

$$
\begin{gathered}
\left\|V_{\Phi} \Phi_{\tau}\right\|_{L_{1 \otimes v_{J}}^{1}} \leq M_{1} \int_{\mathbb{R}^{d}} e^{-\pi \frac{3 z_{1}^{2}}{2 \tau^{2}-2 \tau+5} e^{\pi \frac{(1-2 \tau)^{2} z_{1}^{2}}{\left(2 \tau^{2}-2 \tau+2\right)\left(2 \tau^{2}-2 \tau+5\right)}+\frac{a|1-2 \tau|}{2 \tau^{2}-2 \tau+2}\left|z_{1}\right|} d z_{1}} \\
\times \int_{\mathbb{R}^{d}} e^{-\pi \frac{3 z_{2}^{2}}{2 \tau^{2}-2 \tau+5}} e^{\pi \frac{(1-2 \tau)^{2} z_{2}^{2}}{\left(2 \tau^{2}-2 \tau+2\right)\left(2 \tau^{2}-2 \tau+5\right)}+\frac{a|1-2 \tau|}{2 \tau^{2}-2 \tau+2}\left|z_{2}\right|} d z_{2} \\
=2 M_{1} \int_{\mathbb{R}^{d}} e^{-\pi \frac{3 z_{1}^{2}}{2 \tau^{2}-2 \tau+5}} e^{\pi \frac{(1-2 \tau)^{2} z_{1}^{2}}{\left(2 \tau^{2}-2 \tau+2\right)\left(2 \tau^{2}-2 \tau+5\right)}+\frac{a|1-2 \tau|}{2 \tau^{2}-2 \tau+2}\left|z_{1}\right|} d z_{1} .
\end{gathered}
$$

The integral with respect the variable $z_{1}$ is computed analogously to the one for $\zeta_{1}$ above. The estimate (37) follows by

$$
\left\|\Phi_{\tau}\right\|_{M_{1 \otimes v_{J}}^{1}} \asymp\left\|V_{\Phi} \Phi_{\tau}\right\|_{L_{1 \otimes v_{J}}^{1}} \leq C
$$

as desired.

Proposition 3.1. Under the assumptions of Lemma 3.1, there exists a constant $C>0$ independent of $\tau$ such that

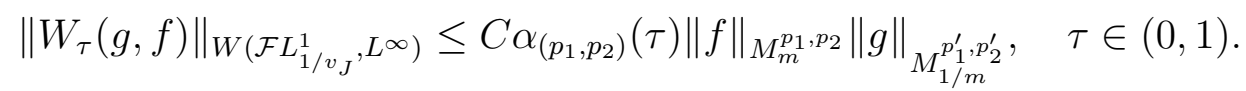


Proof. Changing window in the computation of the STFT as in (17), using Lemmas 2.11, 3.2 and Moyal's formula (13), we have

$$
\begin{aligned}
& \left\|V_{\Phi} W_{\tau}(g, f)\right\|_{L_{z}^{\infty}\left(L_{\zeta, 1 / v_{J}}^{1}\right)} \leq \frac{1}{\left|\left\langle\Phi_{\tau}, \Phi_{\tau}\right\rangle\right|}\left\|\left|V_{\Phi_{\tau}} W_{\tau}(g, f)\right| *\left|V_{\Phi} \Phi_{\tau}\right|\right\|_{L_{z}^{\infty}\left(L_{\zeta, 1 / v_{J}}^{1}\right)} \\
& \leq \frac{1}{\|\varphi\|^{2}\|\varphi\|^{2}}\left\|V_{\Phi_{\tau}} W_{\tau}(g, f)\right\|_{L_{z}^{\infty}\left(L_{\zeta, 1 / v_{J}}^{1}\right)}\left\|V_{\Phi} \Phi_{\tau}\right\|_{L_{1 \otimes v_{J}}^{1}} \\
& \leq C \alpha_{\left(p_{1}, p_{2}\right)}(\tau)\|f\|_{M_{m}^{p_{1}, p_{2}}}\|g\|_{M_{1 / m}^{p_{1}^{\prime}, p_{2}^{\prime}}} \text {. }
\end{aligned}
$$

This completes the proof.

Repeating the pattern of Lemma 3.1 and Proposition 3.1 in the Wiener amalgam space $W\left(\mathcal{F} L_{1 / v_{J}}^{2}, L^{2}\right)\left(\mathbb{R}^{2 d}\right)$, we can state the following.

Proposition 3.2. Let $m \in \mathcal{M}_{v}\left(\mathbb{R}^{2 d}\right)$, $f \in M_{m}^{2}\left(\mathbb{R}^{d}\right)$ and $g \in M_{1 / m}^{2}\left(\mathbb{R}^{d}\right)$. For $\tau \in(0,1)$, the $\tau$-WD $W_{\tau}(g, f)$ is in $W\left(\mathcal{F} L_{1 / v_{J}}^{2}, L^{2}\right)\left(\mathbb{R}^{2 d}\right)$, with the uniform estimate

$$
\left\|W_{\tau}(g, f)\right\|_{W\left(\mathcal{F} L_{1 / v_{J}}^{2}, L^{2}\right)} \leq C\|f\|_{M_{m}^{2}}\|g\|_{M_{1 / m}^{2}}
$$

where the positive constant $C$ is independent of $\tau$.

Proof. First Step. We use Lemma 2.5, Young's Inequality $L^{1} * L^{1} \subset L^{1}$ and the change of variables $\mathcal{B}_{\tau} \eta \rightarrow \eta$, to compute

$$
\begin{aligned}
& \left\|W_{\tau}(g, f)\right\|_{W\left(\mathcal{F} L_{1 / v_{J}}^{2}, L^{2}\right)} \\
& \asymp\left(\int_{\mathbb{R}^{2 d}} \int_{\mathbb{R}^{2 d}}\left|V_{\varphi_{1}} g\left(z+\sqrt{\tau(1-\tau)} \mathcal{A}_{\tau}^{T} \zeta\right)\right|^{2}\left|V_{\varphi_{2}} f\left(z+\sqrt{\tau(1-\tau)} \mathcal{A}_{\tau} \zeta\right)\right|^{2} \frac{1}{v^{2}(J \zeta)} d \zeta d z\right)^{\frac{1}{2}} \\
& \leq C \frac{1}{[\tau(1-\tau)]^{d}}\left(\int_{\mathbb{R}^{2 d}} \int_{\mathbb{R}^{2 d}}\left|V_{\varphi_{1}} g\left(z+\eta-\mathcal{B}_{\tau} \eta\right)\right|^{2}\left|V_{\varphi_{2}} f(z+\eta)\right|^{2} \frac{m^{2}(z+\eta)}{m^{2}\left(z+\eta-\mathcal{B}_{\tau} \eta\right)} d \eta d z\right)^{\frac{1}{2}} \\
& =C \frac{1}{[\tau(1-\tau)]^{d}}\left(\int_{\mathbb{R}^{2 d}}\left(\left|V_{\varphi_{2}} f\right|^{2} m^{2}\right) *\left(\left|V_{\varphi_{1}} g\right|^{2} \frac{1}{m^{2}}\right)\left(\mathcal{B}_{\tau} \eta\right) d \eta\right)^{\frac{1}{2}} \\
& \lesssim\left\|\left|V_{\varphi} f\right|^{2} m^{2}\right\|_{1}\left\|\left|V_{\varphi} g\right|^{2} \frac{1}{m^{2}}\right\|_{1} \\
& \lesssim\|f\|_{M_{m}^{2}}\|g\|_{M_{1 / m}^{2}} .
\end{aligned}
$$

Second Step. Consider now $\Phi \in \mathcal{S}\left(\mathbb{R}^{2 d}\right)$. Then the same pattern as in the proof of Proposition 3.1, with Lemma 2.11 replaced by Lemma 2.12, gives the uniform estimate (39).

The previous issue can be rephrased in terms of modulation spaces as follows (cf. (30)). 
Corollary 3.3. For $\tau \in(0,1), m \in \mathcal{M}_{v}\left(\mathbb{R}^{2 d}\right), f \in M_{m}^{2}\left(\mathbb{R}^{d}\right), g \in M_{1 / m}^{2}\left(\mathbb{R}^{d}\right)$, the $\tau$-WD belongs to $M_{1 / v_{J} \otimes 1}^{2}\left(\mathbb{R}^{2 d}\right)$ with

$$
\left\|W_{\tau}(g, f)\right\|_{M_{1 / v_{J} \otimes 1}^{2}} \leq C\|f\|_{M_{m}^{2}}\|g\|_{M_{1 / m}^{2}}
$$

with $C>0$ independent of $\tau$.

\section{MAin RESUlt}

This section is devoted to the proof of Theorem 4.3. We will start with two preliminary results about $\tau$-pseudodifferential operators acting on modulation spaces and having symbols in $W\left(\mathcal{F} L_{1 / v_{J}}^{1}, L^{\infty}\right)\left(\mathbb{R}^{2 d}\right)$ and $W\left(\mathcal{F} L_{1 / v_{J}}^{2}, L^{2}\right)\left(\mathbb{R}^{2 d}\right)$, respectively. Then, by means of complex interpolation between Wiener amalgam spaces, we shall reach our goal.

Proposition 4.1. Suppose that $m \in \mathcal{M}_{v}\left(\mathbb{R}^{2 d}\right)$ and consider a symbol function $a \in W\left(\mathcal{F} L_{v_{J}}^{\infty}, L^{1}\right)\left(\mathbb{R}^{2 d}\right)$. Then for every $\tau \in(0,1)$, the $\tau$-pseudodifferential operator $\mathrm{Op}_{\tau}(a)$ is bounded on $M_{m}^{p_{1}, p_{2}}\left(\mathbb{R}^{d}\right)$, for every $1 \leq p_{1}, p_{2} \leq \infty$, with

$$
\left\|\mathrm{Op}_{\tau}(a) f\right\|_{M_{m}^{p_{1}, p_{2}}} \leq C \alpha_{\left(p_{1}, p_{2}\right)}(\tau)\|a\|_{W\left(\mathcal{F} L_{v_{J}}^{\infty}, L^{1}\right)}\|f\|_{M_{m}^{p_{1}, p_{2}}}
$$

$(C>0$ does not depend on $\tau)$.

Proof. For every $f \in M_{m}^{p_{1}, p_{2}}\left(\mathbb{R}^{d}\right)$ and $g \in M_{1 / m}^{p_{1}^{\prime}, p_{2}^{\prime}}\left(\mathbb{R}^{d}\right)$, we can write

$$
\left|\left\langle\mathrm{Op}_{\tau}(a) f, g\right\rangle\right|=\left|\left\langle a, W_{\tau}(g, f)\right\rangle\right| \leq\left\|V_{\Phi} a\right\|_{L_{z}^{1}\left(L_{v_{J}, \zeta}^{\infty}\right)}\left\|V_{\Phi} W_{\tau}(g, f)\right\|_{L_{z}^{\infty}\left(L_{1 / v_{J}, \zeta}^{1}\right)} .
$$

Observing that

$$
\left\|W_{\tau}(g, f)\right\|_{W\left(\mathcal{F} L_{1 / v_{J}}^{1}, L^{\infty}\right)} \asymp\left\|V_{\Phi} W_{\tau}(g, f)\right\|_{L_{z}^{\infty}\left(L_{1 / v_{J}, \zeta}^{1}\right)}
$$

and using Proposition 3.1, we conclude the proof.

Proposition 4.2. Let $m \in \mathcal{M}_{v}\left(\mathbb{R}^{2 d}\right), a \in W\left(\mathcal{F} L_{v_{J}}^{2}, L^{2}\right)\left(\mathbb{R}^{2 d}\right)$ and $\tau \in(0,1)$. Then the operator $\mathrm{Op}_{\tau}(a)$ is bounded on $M_{m}^{2}$ with

$$
\left\|\mathrm{Op}_{\tau}(a) f\right\|_{M_{m}^{2}} \leq C\|a\|_{W\left(\mathcal{F} L_{v_{J}}^{2}, L^{2}\right)}\|f\|_{M_{m}^{2}}
$$

where the constant $C>0$ is independent of $\tau$.

Proof. The proof is similar to the one of Proposition 4.1, where Proposition 3.1 is replaced by 3.2 .

Propositions 4.1 and 4.2 are the main ingredients in the proof of Theorem 4.3, which generalizes [10, Theorem 3.1] in the case of $\tau$-pseudodifferential operators. 
Theorem 4.3. Suppose that $1 \leq p, q, r_{1}, r_{2} \leq \infty$ satisfy

$$
q \leq p^{\prime}
$$

and

$$
\max \left\{r_{1}, r_{2}, r_{1}^{\prime}, r_{2}^{\prime}\right\} \leq p
$$

Let $m \in \mathcal{M}_{v}\left(\mathbb{R}^{2 d}\right)$ and $a \in W\left(\mathcal{F} L_{v_{J}}^{p}, L^{q}\right)\left(\mathbb{R}^{2 d}\right)$. For $\tau \in(0,1)$, every $\tau$-pseudodifferential operator $\mathrm{Op}_{\tau}(a)$ is a bounded operator on $M_{m}^{r_{1}, r_{2}}\left(\mathbb{R}^{d}\right)$. Moreover, there exists a constant $C>0$ independent of $\tau$ such that

$$
\left\|\mathrm{Op}_{\tau}(a) f\right\|_{M_{m}^{r_{1}, r_{2}}} \leq C \alpha_{\left(r_{1}, r_{2}\right)}(\tau)\|a\|_{W\left(\mathcal{F} L_{v_{J}}^{p}, L^{q}\right)}\|f\|_{M_{m}^{r_{1}, r_{2}},}, \quad \tau \in(0,1) .
$$

Proof. The key tool is the complex interpolation between Wiener amalgam and modulation spaces. We regard $\mathrm{Op}_{\tau}$ as the bilinear map $(a, f) \mapsto \mathrm{Op}_{\tau}(a) f$. Proposition 4.1 and Proposition 4.2 give the continuity of the $\tau$-pseudodifferential operator $\mathrm{Op}_{\tau}$ on the following function spaces

$$
\begin{gathered}
W\left(\mathcal{F} L_{v_{J}}^{\infty}, L^{1}\right)\left(\mathbb{R}^{2 d}\right) \times M_{m}^{p_{1}, p_{2}}\left(\mathbb{R}^{d}\right) \rightarrow M_{m}^{p_{1}, p_{2}}\left(\mathbb{R}^{d}\right), \\
W\left(\mathcal{F} L_{v_{J}}^{2}, L^{2}\right)\left(\mathbb{R}^{2 d}\right) \times M_{m}^{2}\left(\mathbb{R}^{d}\right) \rightarrow M_{m}^{2}\left(\mathbb{R}^{d}\right),
\end{gathered}
$$

for $1 \leq p_{1}, p_{2} \leq \infty$. Using the complex interpolation between Wiener amalgam and modulation spaces [12], for $\theta \in[0,1]$, we have

$$
\left[W\left(\mathcal{F} L_{v_{J}}^{\infty}, L^{1}\right), W\left(\mathcal{F} L_{v_{J}}^{2}, L^{2}\right)\right]_{\theta}=W\left(\mathcal{F} L_{v_{J}}^{p}, L^{p^{\prime}}\right)
$$

with $2 \leq p \leq \infty$, and $\left[M_{m}^{p_{1}, p_{2}}, M_{m}^{2}\right]_{\theta}=M_{m}^{r_{1}, r_{2}}$, with

$$
\frac{1}{r_{1}}=\frac{1-\theta}{p_{1}}+\frac{\theta}{2}=\frac{1-\theta}{p_{1}}+\frac{1}{p}
$$

and

$$
\frac{1}{r_{2}}=\frac{1-\theta}{p_{2}}+\frac{\theta}{2}=\frac{1-\theta}{p_{2}}+\frac{1}{p}
$$

so that $r_{1}, r_{2} \leq p$. Similarly, we obtain $r_{1}^{\prime}, r_{2}^{\prime} \leq p$, and thus the relation (43). Due to inclusion relations for Wiener amalgam spaces, we relax the assumptions on symbols, so that the symbol a may belong to $W\left(\mathcal{F} L_{v_{J}}^{p}, L^{q}\right)\left(\mathbb{R}^{2 d}\right)$, with $q \leq p^{\prime}$, 
which gives (42). Finally, the norm is provided by

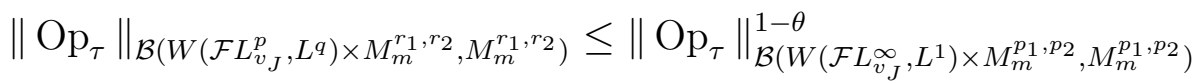

$$
\begin{aligned}
& \times\left\|\mathrm{Op}_{\tau}\right\|_{\mathcal{B}\left(W\left(\mathcal{F} L_{v_{J}}^{2}, L^{2}\right) \times M_{m}^{2}, M_{m}^{2}\right)}^{\theta} \\
& \leq C \frac{1}{\tau^{d(1-\theta)\left(1-\frac{1}{p_{1}}+\frac{1}{p_{2}}\right)}(1-\tau)^{d(1-\theta)\left(1+\frac{1}{p_{1}}-\frac{1}{p_{2}}\right)}} \\
& \leq C \frac{1}{\tau^{d\left(1-\frac{1}{p_{1}}+\frac{1}{p_{2}}\right)}(1-\tau)^{d\left(1+\frac{1}{p_{1}}-\frac{1}{p_{2}}\right)}},
\end{aligned}
$$

since $1-\theta \leq 1$. This concludes the proof.

We finally consider the end-points $\tau=0$ and $\tau=1$, for which the boundedness results stated above do not hold in general. We remark that the modulation space $M^{2}\left(\mathbb{R}^{d}\right)$ is simply the Lebesgue space $L^{2}\left(\mathbb{R}^{d}\right)$. The following example generalizes a 1-dimensional example exhibited by Boulkhemair in [5].

Proposition 4.4. There exists a symbol $a \in W\left(\mathcal{F} L^{\infty}, L^{1}\right)\left(\mathbb{R}^{2 d}\right)$ such that the corresponding Kohn-Nirenberg $\mathrm{Op}_{0}(a)$ and anti-Kohn-Nirenberg $\mathrm{Op}_{1}(a)$ operators are not bounded on $L^{2}\left(\mathbb{R}^{d}\right)$.

Proof. Consider the symbol function

$$
a\left(x_{1}, \ldots, x_{d}, \xi_{1}, \ldots, \xi_{d}\right)=x_{1}^{-1 / 2} \ldots x_{d}^{-1 / 2} \chi_{(0,1]}\left(x_{1}\right) \ldots \chi_{(0,1]}\left(x_{d}\right) e^{-\pi \xi^{2}},
$$

with $\xi^{2}=\xi_{1}^{2}+\cdots+\xi_{d}^{2}$. An easy computation shows that $a \in L^{1}\left(\mathbb{R}^{2 d}\right)=$ $W\left(L^{1}, L^{1}\right)\left(\mathbb{R}^{2 d}\right) \subset W\left(\mathcal{F} L^{\infty}, L^{1}\right)\left(\mathbb{R}^{2 d}\right)$. Let us show that the Kohn-Niremberg $\mathrm{Op}_{0}(a)$ is unbounded on $L^{2}\left(\mathbb{R}^{d}\right)$. Consider the Gaussian function $f(t)=e^{\pi t^{2}} \in$ $L^{2}\left(\mathbb{R}^{d}\right)$, then $\mathrm{Op}_{0}(a) f \notin L^{2}\left(\mathbb{R}^{d}\right)$. Indeed, by a tensor product argument, we reduce to compute the following one-dimensional integral:

$$
\int_{\mathbb{R}} e^{2 \pi i x \xi} x^{-1 / 2} \chi_{(0,1]}(x) e^{-\pi \xi^{2}} e^{-\pi \xi^{2}} d \xi=\frac{1}{\sqrt{2}} x^{-1 / 2} \chi_{(0,1]}(x) e^{-\pi \frac{x^{2}}{2}},
$$

whose result is a function that does not belong to $L^{2}(\mathbb{R})$.

To prove that the anti-Kohn-Nirenberg operator $\mathrm{Op}_{1}(a)$, where $a$ is defined in (47), is unbounded on $L^{2}\left(\mathbb{R}^{d}\right)$, it is sufficient to observe that its adjoint operator is the Kohn-Niremberg one: $\left(\mathrm{Op}_{1}(a)\right)^{*}=\mathrm{Op}_{0}(a)$, as detailed below:

$$
\left\langle\mathrm{Op}_{1}(a) f, g\right\rangle=\left\langle a, \mathcal{R}^{*}(g, f)\right\rangle=\langle a, \overline{\mathcal{R}(f, g)}\rangle=\langle\mathcal{R}(f, g), \bar{a}\rangle=\left\langle f, \mathrm{Op}_{0}(a) g\right\rangle .
$$

This proves our claim. 


\section{REMARKS ON BOUNDEDNESS RESULTS FOR SYMBOLS IN MODULATION SPACES}

We address this section to study the boundedness results for $\tau$-pseudodifferential operators with symbols in weighted modulation spaces. Recall (cf. [19] and [31, Remark 1.5]) that for every choice $\tau_{1}, \tau_{2} \in[0,1], a_{1}, a_{2} \in \mathcal{S}^{\prime}\left(\mathbb{R}^{2 d}\right)$,

$$
\mathrm{Op}_{\tau_{1}}\left(a_{1}\right)=\mathrm{Op}_{\tau_{2}}\left(a_{2}\right) \Leftrightarrow \widehat{a_{2}}\left(\xi_{1}, \xi_{2}\right)=e^{-2 \pi i\left(\tau_{2}-\tau_{1}\right) \xi_{1} \xi_{2}} \widehat{a_{1}}\left(\xi_{1}, \xi_{2}\right) .
$$

For $t>0$ define $H_{t}(x, \xi)=e^{2 \pi i t x \xi}$ and observe that

$$
\mathcal{F} H_{t}\left(\zeta_{1}, \zeta_{2}\right)=\frac{1}{t^{d}} e^{-2 \pi i \frac{1}{t} \zeta_{1} \zeta_{2}}
$$

So, for $\tau_{1} \neq \tau_{2}$, by (49),

$$
a_{2}(x, \xi)=\frac{1}{\left|\tau_{1}-\tau_{2}\right|^{d}} e^{2 \pi i\left(\tau_{2}-\tau_{1}\right) \Psi} * a_{1}(x, \xi),
$$

where $\Psi(x, \xi)=x \xi$. Toft in [31, Proposition 1.2 (5)] proved that the mapping $a \mapsto T_{\Phi} a=e^{2 \pi i \Phi} * a$ is a homeomorphism on $M^{p, q}\left(\mathbb{R}^{2 d}\right), 1 \leq p, q \leq \infty$. This implies that results for Weyl operators with symbols in modulation spaces are still true for any $\tau$-operator. The main goal of this section is to show uniform estimates for $\tau$ operators with symbol in weighted modulation spaces. Following the pattern of the previous section, we first compute the norm of the $\tau$-WD in weighted modulation spaces.

The next Proposition extends the sufficient conditions of [6, Theorem 1.1] in the case of $\tau$-Wigner distributions.

Proposition 5.1. Assume that $p_{1}, p_{2}, q_{1}, q_{2}, p, q \in[0,1]$ satisfy

$$
p_{i}, q_{i} \leq q, \quad i=1,2,
$$

and

$$
\frac{1}{p_{1}}+\frac{1}{p_{2}} \geq \frac{1}{p}+\frac{1}{q}, \quad \frac{1}{q_{1}}+\frac{1}{q_{2}} \geq \frac{1}{p}+\frac{1}{q} .
$$

Consider $m \in \mathcal{M}_{v}, f \in M_{m}^{p_{1}, q_{1}}\left(\mathbb{R}^{d}\right)$ and $g \in M_{1 / m}^{p_{2}, q_{2}}\left(\mathbb{R}^{d}\right)$. Then, for any $\tau \in[0,1]$, $W_{\tau}(g, f) \in M_{1 \otimes 1 / v_{J}}^{p, q}\left(\mathbb{R}^{2 d}\right)$. Furthermore, there exists a constant $C>0$, independent of $\tau \in[0,1]$, such that

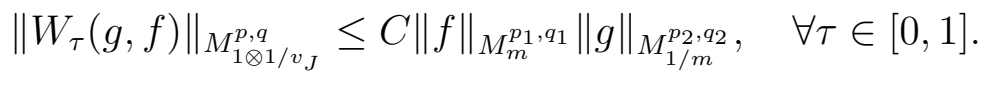

Proof. We separate the proof in three cases: $\tau \in(0,1), \tau=0$ and $\tau=1$.

Case $\tau \in(0,1)$. Assume $p \leq q<\infty$. Making the change of variables $z+$ 
$\sqrt{\tau(1-\tau)} \mathcal{A}_{\tau} \zeta=y$ and using item (iv) of Lemma 2.1, the integral with respect the variable $z$ becomes

$$
\begin{aligned}
\| & W_{\tau}(g, f) \|_{M_{1 \otimes 1 / v_{J}}^{p, q}} \\
& =\left(\int_{\mathbb{R}^{2 d}}\left(\int_{\mathbb{R}^{2 d}}\left|V_{\varphi_{1}} g\left(z+\sqrt{\tau(1-\tau)} \mathcal{A}_{\tau}^{T} \zeta\right)\right|^{p}\left|V_{\varphi_{2}} f\left(z+\sqrt{\tau(1-\tau)} \mathcal{A}_{\tau} \zeta\right)\right|^{p} d z\right)^{\frac{q}{p}} \frac{1}{v^{q}(J \zeta)} d \zeta\right)^{\frac{1}{q}} \\
& =\left(\int_{\mathbb{R}^{2 d}}\left(\int_{\mathbb{R}^{2 d}}\left|V_{\varphi_{1}} g(y-J \zeta)\right|^{p}\left|V_{\varphi_{2}} f(y)\right|^{p} \frac{1}{v^{p}(J \zeta)} d y\right)^{\frac{q}{p}} d \zeta\right)^{\frac{1}{q}} \\
& \leq C\left(\int_{\mathbb{R}^{2 d}}\left(\int_{\mathbb{R}^{2 d}}\left|V_{\varphi_{1}} g(y-J \zeta)\right|^{p}\left|V_{\varphi_{2}} f(y)\right|^{p} \frac{m^{p}(y)}{m^{p}(y-J \zeta)} d y\right)^{\frac{q}{p}} d \zeta\right)^{\frac{1}{q}} \\
& =C\left(\int_{\mathbb{R}^{2 d}}\left(\left(\left|\mathcal{I} V_{\varphi_{1}} g\right|^{p} \frac{1}{m^{p}}\right) *\left(\left|V_{\varphi_{2}} f\right|^{p} m^{p}\right)(J \zeta)\right)^{\frac{q}{p}} d \zeta\right)^{\frac{1}{q}} \\
& =C\left\|\mid\left(\left.\mathcal{I} V_{\varphi_{1}} g\right|^{p} \frac{1}{m^{p}}\right) *\left(\left|V_{\varphi_{2}} f\right|^{p} m^{p}\right)\right\|_{L^{q / p}}^{1 / p},
\end{aligned}
$$

where $\mathcal{I}$ is the reflection operator. The rest goes exactly as in the proof of Theorem 3.1 in [6], obtaining the estimate

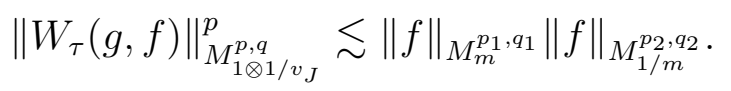

Using Lemma 3.2, there exists a positive constant $C$ independent of $\tau \in(0,1)$ such that

$\left\|W_{\tau}(g, f)\right\|_{M_{1 \otimes 1 / v_{J}}^{p, q}} \leq \frac{1}{\left|\left\langle\Phi_{\tau}, \Phi_{\tau}\right\rangle\right|}\left\|V_{\Phi_{\tau}} W_{\tau}(g, f) * V_{\Phi} \Phi_{\tau}\right\|_{L_{1 \otimes 1 / v_{J}}^{p, q}} \leq C\|f\|_{M_{m}^{p_{1}, q_{1}}}\|g\|_{M_{1 / m}^{p_{2}, q_{2}}}$

concluding the proof for $\tau \in(0,1), p \leq q<\infty$.

Assume $p=q=\infty$. We have 


$$
\begin{aligned}
\left\|W_{\tau}(g, f)\right\|_{M_{1 \otimes 1 / v}^{\infty}} & =\sup _{z, \zeta \in \mathbb{R}^{2 d}}\left|V_{\varphi_{1}} g\left(z+\sqrt{\tau(1-\tau)} \mathcal{A}_{\tau}^{T} \zeta\right)\right|\left|V_{\varphi_{2}} f\left(z+\sqrt{\tau(1-\tau)} \mathcal{A}_{\tau} \zeta\right)\right| \frac{1}{v(J \zeta)} \\
& =\sup _{\zeta \in \mathbb{R}^{2 d}} \sup _{z \in \mathbb{R}^{2 d}}\left|V_{\varphi_{1}} g(z-J \zeta)\right|\left|V_{\varphi_{2}} f(z)\right| \frac{1}{v(J \zeta)} \\
& \leq C \sup _{\zeta \in \mathbb{R}^{2 d}} \sup _{z \in \mathbb{R}^{2 d}}\left|V_{\varphi_{1}} g(z-J \zeta)\right|\left|V_{\varphi_{2}} f(z)\right| \frac{m(z)}{m(z-J \zeta)} \\
& =C \sup _{z \in \mathbb{R}^{2 d}}\left|V_{\varphi_{2}} f(z)\right| m(z)\left(\sup _{\zeta \in \mathbb{R}^{2 d}}\left|V_{\varphi_{1}} g(z-J \zeta)\right| \frac{1}{m(z-J \zeta)}\right) \\
& =C\left\|\left|V_{\varphi_{2}} f\right| m\right\|_{L^{\infty}}\left\|\left|V_{\varphi_{2}} g\right| \frac{1}{m}\right\|_{L^{\infty}} \\
& =\|f\|_{M_{1 / v}^{\infty}}\|g\|_{M_{v}^{\infty}} \\
& \leq\|f\|_{M_{1 / v}^{p_{1}, q_{1}}}\|g\|_{M_{v}^{p_{2}, q_{2}}}
\end{aligned}
$$

for every $1 \leq p_{i}, q_{i} \leq \infty$. The conclusion follows again by Lemma 3.2 and Young's inequality.

Assume $p>q$. Using the inclusion relations for modulation spaces, we majorize

$$
\left\|W_{\tau}(g, f)\right\|_{M_{1 \otimes 1 / v_{J}}^{p, q}} \leq\left\|W_{\tau}(g, f)\right\|_{M_{1 \otimes 1 / v_{J}}^{q, q}} \leq C\|f\|_{M_{m}^{p_{1}, q_{1}}}\|g\|_{M_{1 / m}^{p_{2}, q_{2}}}
$$

for every $1 \leq p_{i}, q_{i} \leq q, i=1,2$.

Case $\tau=0$. In this case, we obtain at once a uniform estimate. Indeed, using Lemma 2.6,

$$
\begin{aligned}
\left\|W_{0}(g, f)\right\|_{M_{1 \otimes 1 / v_{J}}^{p, q}} & \\
\quad= & \left(\int_{\mathbb{R}^{d}}\left(\int_{\mathbb{R}^{d}}\left|V_{\varphi_{1}} g\left(z_{1}, z_{2}+\zeta_{1}\right)\right|^{p}\left|V_{\varphi_{2}} f\left(z_{1}+\zeta_{2}, z_{2}\right)\right|^{p} d z_{1} d z_{2}\right)^{q / p} \frac{1}{v_{J}^{q}(\zeta)} d \zeta_{1} d \zeta_{2}\right)^{1 / q} \\
& =\left(\int_{\mathbb{R}^{d}}\left(\int_{\mathbb{R}^{d}}\left|V_{\varphi_{1}} g\left(z_{1}-\zeta_{2}, z_{2}+\zeta_{1}\right)\right|^{p}\left|V_{\varphi_{2}} f\left(z_{1}, z_{2}\right)\right|^{p} \frac{1}{v^{p}(J \zeta)} d z_{1} d z_{2}\right)^{q / p} d \zeta_{1} d \zeta_{2}\right)^{1 / q} \\
& \leq C\left(\int_{\mathbb{R}^{d}}\left(\int_{\mathbb{R}^{d}}\left|V_{\varphi_{1}} g(z-J \zeta)\right|^{p}\left|V_{\varphi_{2}} f(z)\right|^{p} \frac{m^{p}(z)}{m^{p}(x-J \zeta)} d z\right)^{q / p} d \zeta\right)^{1 / q} \\
& =C\left\|\left(\left|V_{\varphi_{1}} g\right|^{p}\left(1 / m^{p}\right)\right) *\left(\left|V_{\varphi_{2}} f\right|^{p} m^{p}\right)\right\|_{L^{q / p}} .
\end{aligned}
$$

Then we proceed as in Case $\tau \in(0,1)$.

Case $\tau=1$. The proof is analogous to the one of Case $\tau=0$. We are done.

The boundedness results for $\tau$-WDs transfer to $\tau$-pseudodifferential operators as follows. 
Theorem 5.1. Let $1 \leq p_{1}, p_{2}, q_{1}, q_{2}, p \leq \infty$ be indices such that

$$
p_{1}, p_{2}^{\prime}, q_{1}, q_{2}^{\prime} \leq q^{\prime}
$$

and

$$
\frac{1}{p_{1}}+\frac{1}{p_{2}^{\prime}} \geq \frac{1}{p^{\prime}}+\frac{1}{q^{\prime}}, \quad \frac{1}{q_{1}}+\frac{1}{q_{2}^{\prime}} \geq \frac{1}{p^{\prime}}+\frac{1}{q^{\prime}}
$$

Let $m \in \mathcal{M}_{v}\left(\mathbb{R}^{2 d}\right)$. For every $\tau \in[0,1]$, the $\tau$-pseudodifferential operator $\operatorname{Op}_{\tau}(a)$, with symbol $a \in M_{1 \otimes v_{J}}^{p, q}\left(\mathbb{R}^{2 d}\right)$, is a bounded operator from $M_{m}^{p_{1}, q_{1}}\left(\mathbb{R}^{d}\right)$ to $M_{m}^{p_{2}, q_{2}}\left(\mathbb{R}^{d}\right)$, with

$$
\left\|\mathrm{Op}_{\tau}(a) f\right\|_{M_{m}^{p_{2}, q_{2}}} \leq C\|a\|_{M_{1 \otimes v_{J}}^{p, q}}\|f\|_{M_{m}^{p_{1}, q_{1}}}
$$

and $C>0$ is independent of $\tau$.

Proof. If $f \in M_{m}^{p_{1}, q_{1}}\left(\mathbb{R}^{d}\right)$ and $g \in M_{1 / m}^{p_{2}^{\prime}, q_{2}^{\prime}}\left(\mathbb{R}^{d}\right)$, then $W_{\tau}(g, f) \in M_{1 \otimes \frac{1}{v_{J}}}^{p^{\prime}, q^{\prime}}\left(\mathbb{R}^{2 d}\right)$, by Proposition 5.1, provided that (53) and (54) hold. Thereby there exists a positive constant $C$ such that for any $\tau \in[0,1]$,

$$
\begin{aligned}
\left|\left\langle\mathrm{Op}_{\tau}(a) f, g\right\rangle\right| & =\mid\left\langle a, W_{\tau}(g, f)\right| \\
& \leq C\|a\|_{M_{1 \otimes v_{J}}^{p, q}}\|f\|_{M_{m}^{p_{1}, q_{1}}}\|g\|_{M_{1 / m}^{p_{2}^{\prime}, q_{2}^{\prime}}},
\end{aligned}
$$

as desired.

\section{REFERENCES}

[1] A. Benedek and R. Panzone. The space $L^{p}$, with mixed norm. Duke Math. J., 28:301-324, 1961.

[2] Á. Bényi, K. Gröchenig, C. Heil, and K. Okoudjou. Modulation spaces and a class of bounded multilinear pseudodifferential operators. J. Operator Theory, 54(2):387-399, 2005.

[3] P. Boggiatto, G. De Donno, and A. Oliaro. Time-frequency representations of Wigner type and pseudo-differential operators. Trans. Amer. Math. Soc., 362(9):4955-4981, 2010.

[4] M. Born and P. Jordan. Zur quantenmechanik. Zeitschrift für Physik, 34(1):858-888, Dec 1925.

[5] A. Boulkhemair. $L^{2}$ estimates for pseudodifferential operators. Ann. Scuola Norm. Sup. Pisa Cl. Sci. (4), 22(1):155-183, 1995.

[6] E. Cordero and F. Nicola. Sharp integral bounds for Wigner distributions. International Mathematics Research Notices, 2016(00):1-29.

[7] E. Cordero, A. Tabacco and P. Wahlberg. Schrödinger-type propagators, pseudodifferential operators and modulation spaces. J. London Math. Soc., 88(2):375-395, 2013.

[8] E. Cordero, J. Toft, and P. Wahlberg. Sharp results for the Weyl product on modulation spaces. J. Funct. Anal., 267(8):3016-3057, 2014.

[9] M. A. de Gosson. Symplectic methods in harmonic analysis and in mathematical physics, volume 7 of Pseudo-Differential Operators. Theory and Applications. Birkhäuser/Springer Basel AG, Basel, 2011. 
[10] L. D'Elia and S. I. Trapasso. Boundedness of pseudodifferential operators with symbols in wiener amalgam spaces on modulation spaces. Journal of Pseudo-Differential Operators and Applications, 1-10, 2017.

[11] H. G. Feichtinger. Banach convolution algebras of Wiener type. In Functions, series, operators, Vol. I, II (Budapest, 1980), volume 35 of Colloq. Math. Soc. János Bolyai, pages 509-524. North-Holland, Amsterdam, 1983.

[12] H. G. Feichtinger. Banach spaces of distributions of Wiener's type and interpolation. In Functional analysis and approximation (Oberwolfach, 1980), volume 60 of Internat. Ser. Numer. Math., pages 153-165. Birkhäuser, Basel-Boston, Mass., 1981.

[13] H. G. Feichtinger. Generalized amalgams, with applications to Fourier transform. Canad. J. Math., 42(3):395-409, 1990.

[14] K. Gröchenig. Foundations of time-frequency analysis. Applied and Numerical Harmonic Analysis. Birkhäuser Boston, Inc., Boston, MA, 2001.

[15] K. Gröchenig. Weight functions in time-frequency analysis. Pseudo-differential operators: partial differential equations and time-frequency analysis, volume 52 of Fields Inst. Commun., pages 343-366. Amer. Math. Soc., Providence, RI, 2007.

[16] K. Gröchenig and C. Heil. Modulation spaces and pseudodifferential operators. Integral Equations Operator Theory, 34(4):439-457, 1999.

[17] K. Gröchenig and C. Heil. Counterexamples for boundedness of pseudodifferential operators. Osaka J. Math., 41(3):681-691, 2004.

[18] K. Gröchenig and T. Strohmer. Pseudodifferential operators on locally compact abelian groups and Sjöstrand's symbol class. J. Reine Angew. Math., 613:121-146, 2007.

[19] L. Hörmander. The analysis of linear partial differential operators. III, volume 274 of Grundlehren der Mathematischen Wissenschaften [Fundamental Principles of Mathematical Sciences]. Springer-Verlag, Berlin, 1994. Pseudo-differential operators, Corrected reprint of the 1985 original.

[20] J. J. Kohn and L. Nirenberg. An algebra of pseudo-differential operators. Comm. Pure Appl. Math., 18:269-305, 1965.

[21] D. Labate. Pseudodifferential operators on modulation spaces. J. Math. Anal. Appl., 262:242$255,2001$.

[22] M. Ruzhansky, B. Wang, and H. Zhang. Global well-posedness and scattering for the fourth order nonlinear Schrödinger equations with small data in modulation and Sobolev spaces, J. Math. Pures Appl., 105(9):31-65, 2016.

[23] M. A. Shubin. Pseudodifferential operators and spectral theory. Springer-Verlag, Berlin, second edition, 2001. Translated from the 1978 Russian original by Stig I. Andersson.

[24] J. Sjöstrand. An algebra of pseudodifferential operators. Math. Res. Lett., 1(2):185-192, 1994.

[25] J. Sjöstrand. Wiener type algebras of pseudodifferential operators, Séminaire sur les Équations aux Dérivées Partielles, 1994-1995, École Polytech., Palaiseau, 1995, pp. Exp. No. IV, 21. MR 1362552

[26] E. M. Stein. Harmonic analysis: real-variable methods, orthogonality, and oscillatory integrals, volume 43 of Princeton Mathematical Series. Princeton University Press, Princeton, NJ, 1993. With the assistance of Timothy S. Murphy, Monographs in Harmonic Analysis, III.

[27] K. Tachizawa. The boundedness of pseudo-differential operators on modulation spaces. Math. Nachr., 168:263-277, 1994.

[28] M. E. Taylor. Pseudodifferential operators, volume 34 of Princeton Mathematical Series. Princeton University Press, Princeton, N.J., 1981. 
[29] N. Teofanov. Bilinear Localization Operators on Modulation Spaces, J. Funct. Spaces, 2018. https://doi.org/10.1155/2018/7560870

[30] N. Teofanov. Continuity and Schatten-von Neumann properties for localization operators on modulation spaces, Mediterr. J. Math., 13(2):745-758, 2016.

[31] J. Toft. Continuity properties for modulation spaces, with applications to pseudo-differential calculus. I. J. Funct. Anal., 207(2):399-429, 2004.

[32] J. Toft. Continuity properties for modulation spaces, with applications to pseudo-differential calculus. II, Ann. Global Anal. Geom., 26 (1):73-106, 2004.

[33] F. Trèves. Introduction to pseudodifferential and Fourier integral operators. Vol. 2. Plenum Press, New York-London, 1980. Fourier integral operators, The University Series in Mathematics.

[34] M. W. Wong. Weyl transforms. Universitext. Springer-Verlag, New York, 1998.

Dipartimento di Matematica, Universitì di Torino, via Carlo Alberto 10, 10123 TORINO, ITALY

E-mail address: elena.cordero@unito.it

Dipartimento di Matematica, Universitì di Torino, via Carlo Alberto 10, 10123 TORINO, ITALY

E-mail address: lorenza.delia@unito.it

Dipartimento di Scienze Matematiche, Politecnico di Torino, Corso Duca degli Abruzzi 24, 10129 Torino, Italy

E-mail address: salvatore.trapasso@polito.it 